\title{
Asymptotically flat structure of hypergravity in three spacetime dimensions
}

\author{
Oscar Fuentealba, ${ }^{a, b}$ Javier Matulich $^{a}$ and Ricardo Troncoso, ${ }^{a}$ \\ ${ }^{a}$ Centro de Estudios Cientificos (CECs), \\ Av. Arturo Prat 514, Valdivia, Chile \\ ${ }^{b}$ Departamento de Fúsica, Universidad de Concepción, \\ Casilla 160-C, Concepción, Chile \\ E-mail: fuentealba@cecs.cl, matulich@cecs.cl, troncoso@cecs.cl
}

ABSTRACT: The asymptotic structure of three-dimensional hypergravity without cosmological constant is analyzed. In the case of gravity minimally coupled to a spin- $5 / 2$ field, a consistent set of boundary conditions is proposed, being wide enough so as to accommodate a generic choice of chemical potentials associated to the global charges. The algebra of the canonical generators of the asymptotic symmetries is given by a hypersymmetric nonlinear extension of $\mathrm{BMS}_{3}$. It is shown that the asymptotic symmetry algebra can be recovered from a subset of a suitable limit of the direct sum of the $\mathrm{W}_{(2,4)}$ algebra with its hypersymmetric extension. The presence of hypersymmetry generators allows to construct bounds for the energy, which turn out to be nonlinear and saturate for spacetimes that admit globally-defined "Killing vector-spinors". The null orbifold or Minkowski spacetime can then be seen as the corresponding ground state in the case of fermions that fulfill periodic or antiperiodic boundary conditions, respectively. The hypergravity theory is also explicitly extended so as to admit parity-odd terms in the action. It is then shown that the asymptotic symmetry algebra includes an additional central charge, being proportional to the coupling of the Lorentz-Chern-Simons form. The generalization of these results in the case of gravity minimally coupled to arbitrary half-integer spin fields is also carried out. The hypersymmetry bounds are found to be given by a suitable polynomial of degree $s+\frac{1}{2}$ in the energy, where $s$ is the spin of the fermionic generators.

KeYwords: Higher Spin Symmetry, Conformal and W Symmetry, Gauge-gravity correspondence, Classical Theories of Gravity

ArXiv EPrint: 1508.04663 


\section{Contents}

1 Introduction 1

2 General Relativity minimally coupled to a spin-5/2 field 2

3 Unbroken hypersymmetries: Killing vector-spinors 4

3.1 Cosmological spacetimes and solutions with conical defects 4

4 Asymptotically flat behaviour and the hyper-BMS ${ }_{3}$ algebra 6

4.1 Flat limit of the asymptotic symmetry algebra from the case of negative cosmological constant

5 Hypersymmetry bounds

6 Hypergravity reloaded

7 General Relativity minimally coupled to half-integer spin fields $\quad \mathbf{1 5}$

$\begin{array}{lll}7.1 & \text { Killing tensor-spinors } & 16\end{array}$

$\begin{array}{lll}7.2 & \text { Asymptotically flat structure and hypersymmetry bounds } & 17\end{array}$

8 Final remarks $\quad 20$

$\begin{array}{ll}\text { A Conventions } & 24\end{array}$

B Killing vector-spinors from an alternative approach $\quad 24$

C Hyper-Poincaré algebra with fermionic generators of spin $n+\frac{1}{2} \quad 26$

D Asymptotic hypersymmetry algebra $\quad \mathbf{2 6}$

D.1 Spin-3/2 fields (supergravity) 26

D.2 Spin- $7 / 2$ fields 27

D.3 Spin-9/2 fields 29

\section{Introduction}

It has been shown that the inconsistencies arising in the minimal coupling of a massless spin$5 / 2$ field to General Relativity [1-4] can be successfully surmounted in three-dimensional spacetimes [5]. This theory is known as hypergravity, and it has been recently reformulated as a Chern-Simons theory of a new extension of the Poincaré group with fermionic generators of spin $3 / 2$ [6]. In the case of negative cosmological constant, additional spin- 4 fields are required by consistency [7-9], and it can be seen that the anticommutator of the 
generators of the asymptotic hypersymmetries, associated to fermionic spin- $3 / 2$ parameters, leads to interesting nonlinear bounds for the bosonic global charges of spin 2 and 4 [9]. The bounds saturate provided the bosonic configurations admit globally-defined "Killing vector-spinors". One of the main purposes of this paper is to show how these results extend to the case of asymptotically flat spacetimes in hypergravity, also in the case of arbitrary half-integer spin fields. In the next section we briefly summarize the formulation of hypergravity as a Chern-Simons theory for the hyper-Poincaré group in the simplest case of fermionic spin-5/2 fields, while section 3 is devoted to explore the global hypersymmetry properties of cosmological spacetimes and solutions with conical defects. In the case of fermions that fulfill periodic boundary conditions, it is shown that the null orbifold possesses a single constant Killing vector-spinor. Analogously, for antiperiodic boundary conditions, Minkowski spacetime is singled out as the maximally (hyper)symmetric configuration, and the explicit expression of the globally-defined Killing vector-spinors is found. The asymptotically flat structure of hypergravity in three spacetime dimensions is analyzed in section 4, where a precise set of boundary conditions that includes "chemical potentials" associated to the global charges is proposed. The algebra of the canonical generators of the asymptotic symmetries is found to be given by a suitable hypersymmetric nonlinear extension of the $\mathrm{BMS}_{3}$ algebra. It is also shown that this algebra corresponds to a subset of a suitable Inönü-Wigner contraction of the direct sum of the $\mathrm{W}_{(2,4)}$ algebra with its hypersymmetric extension $\mathrm{W}_{\left(2, \frac{5}{2}, 4\right)}$. The hypersymmetry bounds that arise from the anticommutator of the fermionic generators are found to be nonlinear, and are shown to saturate for spacetimes that admit unbroken hypersymmetries, like the ones aforementioned. This is explicitly carried out in section 5. In section 6 , the previous analysis is performed in the case of an extension of the hypergravity theory that includes additional parity-odd terms in the action. It is found that the asymptotic symmetry algebra admits an additional central charge along the Virasoro subgroup. The results are then extended to the case of General Relativity minimally coupled to half-integer spin fields in section 7, including the asymptotically flat structure, and the explicit expression of the Killing tensor-spinors. The hypersymmetry bounds are shown to be described by a polynomial of degree $s+1 / 2$ in the energy, where $s$ is the spin of the fermionic generators. We conclude in section 8 with some final remarks, including the extension of these results to the case of hypergravity with additional parity-odd terms and fermions of arbitrary half-integer spin. The coupling of additional spin-4 fields is also addressed. Appendix A is devoted to our conventions, and in appendix B, an alternative interesting form to obtain the explicit form of the Killing vector-spinors is presented. The general form of the hyper-Poincaré algebra is discussed in appendix $\mathrm{C}$, while appendix $\mathrm{D}$ includes the asymptotic hypersymmetry algebra in the case of fermionic fields of spin $3 / 2$ (supergravity), as well as for fields of spin $7 / 2$ and $9 / 2$.

\section{General Relativity minimally coupled to a spin-5/2 field}

It has been recently shown that the hypergravity theory of Aragone and Deser [5] can be reformulated as a gauge theory of a suitable extension of the Poincaré group with fermionic spin-3/2 generators [6]. The action is described by a Chern-Simons form, so 
that the dreibein, the (dualized) spin connection, and the spin-5/2 field correspond to the components of a gauge field given by

$$
A=e^{a} P_{a}+\omega^{a} J_{a}+\psi_{a}^{\alpha} Q_{\alpha}^{a},
$$

that takes values in the hyper-Poincaré algebra, being spanned by the set $\left\{P_{a}, J_{a}, Q_{\alpha}^{a}\right\}$. The fermionic fields and generators are assumed to be $\Gamma$-traceless, i. e., $\Gamma^{a} \psi_{a}=0$, and $Q^{a} \Gamma_{a}=0$, so that the nonvanishing (anti)commutation rules read

$$
\begin{aligned}
{\left[J_{a}, J_{b}\right] } & =\varepsilon_{a b c} J^{c}, \quad\left[J_{a}, P_{b}\right]=\varepsilon_{a b c} P^{c}, \\
{\left[J_{a}, Q_{\alpha b}\right] } & =\frac{1}{2}\left(\Gamma_{a}\right)_{\alpha}^{\beta} Q_{\beta b}+\varepsilon_{a b c} Q_{\alpha}^{c}, \\
\left\{Q_{\alpha}^{a}, Q_{\beta}^{b}\right\} & =-\frac{2}{3}\left(C \Gamma^{c}\right)_{\alpha \beta} P_{c} \eta^{a b}+\frac{5}{6} \varepsilon^{a b c} C_{\alpha \beta} P_{c}+\frac{1}{6}\left(C \Gamma^{(a}\right)_{\alpha \beta} P^{b)},
\end{aligned}
$$

where $C$ stands for the charge conjugation matrix. The Majorana conjugate then reads $\bar{\psi}_{\alpha a}=\psi_{a}^{\beta} C_{\beta \alpha}$. Since the algebra admits an invariant bilinear form, whose only nonvanishing components are given by

$$
\left\langle J_{a}, P_{b}\right\rangle=\eta_{a b}, \quad\left\langle Q_{\alpha}^{a}, Q_{\beta}^{b}\right\rangle=\frac{2}{3} C_{\alpha \beta} \eta^{a b}-\frac{1}{3} \varepsilon^{a b c}\left(C \Gamma_{c}\right)_{\alpha \beta},
$$

the action can be written as

$$
I[A]=\frac{k}{4 \pi} \int\left\langle A d A+\frac{2}{3} A^{3}\right\rangle,
$$

which up to a surface term reduces to

$$
I=\frac{k}{4 \pi} \int 2 R^{a} e_{a}+i \bar{\psi}_{a} D \psi^{a} .
$$

Here $R^{a}=d \omega^{a}+\frac{1}{2} \varepsilon^{a b c} \omega_{b} \omega_{c}$ is the dual of the curvature two-form, and since the fermionic field is $\Gamma$-traceless, its Lorentz covariant derivative fulfills

$$
\begin{aligned}
D \psi^{a} & =d \psi^{a}+\frac{1}{2} \omega^{b} \Gamma_{b} \psi^{a}+\varepsilon^{a b c} \omega_{b} \psi_{c} \\
& =d \psi^{a}+\frac{3}{2} \omega^{b} \Gamma_{b} \psi^{a}-\omega_{b} \Gamma^{a} \psi^{b} .
\end{aligned}
$$

The field equations are then given by $F=d A+A^{2}=0$, whose components read

$$
R^{a}=0, \quad T^{a}=\frac{3}{4} i \bar{\psi}_{b} \Gamma^{a} \psi^{b}, \quad D \psi^{a}=0,
$$

where $T^{a}=D e^{a}$ corresponds to the torsion two-form.

Therefore, by construction, the action changes by a boundary term under local hypersymmetry transformations spanned by $\delta A=d A+[A, \lambda]$, with $\lambda=\epsilon_{a}^{\alpha} Q_{\alpha}^{a}$, so that the transformation law of the fields reduces to

$$
\delta e^{a}=\frac{3}{2} i \bar{\epsilon}_{b} \Gamma^{a} \psi^{b}, \quad \delta \omega^{a}=0, \quad \delta \psi^{a}=D \epsilon^{a} .
$$

Note that the transformation rules of the fields in [5] agree with the ones in (2.8), on-shell. 


\section{Unbroken hypersymmetries: Killing vector-spinors}

It is interesting to explore the set of bosonic solutions that possess unbroken global hypersymmetries. According to the transformation rules of the fields in (2.8), this class of configurations has to fulfill the following Killing vector-spinor equation:

$$
d \epsilon^{a}+\frac{1}{2} \omega^{b} \Gamma_{b} \epsilon^{a}+\varepsilon^{a b c} \omega_{b} \epsilon_{c}=0,
$$

where the spin-3/2 parameter $\epsilon^{a}$ is $\Gamma$-traceless.

As it follows from the field equations (2.7), the spin connection is locally flat, and it can then be written as $\omega=\omega^{a} J_{a}=g^{-1} d g$, with $g=e^{\lambda^{a} J_{a}}$. Therefore, the general solution of the Killing vector-spinor equation (3.1) is given by

$$
\epsilon_{a}^{\alpha}=\left(g_{S}^{-1}\right)_{\beta}^{\alpha}\left(g_{V}\right)_{a}^{b} \eta_{b}^{\beta},
$$

where $\eta_{b}^{\beta}$ is a $\Gamma$-traceless constant vector-spinor. Here, $g_{S}$ and $g_{V}$ stand for the same group element $g$, but expressed in the spinor and the vector (adjoint) representations, respectively. Since the generators of the Lorentz group in the spinor and vector representations are given by $\left(J_{a}\right)_{\beta}^{\alpha}=\frac{1}{2}\left(\Gamma_{a}\right)_{\beta}^{\alpha}$, and $\left(J_{a}\right)_{b c}=-\varepsilon_{a b c}$, they explicitly read

$$
\left(g_{S}\right)_{\beta}^{\alpha}=\exp \left[\frac{1}{2} \lambda^{a}\left(\Gamma_{a}\right)_{\beta}^{\alpha}\right], \quad\left(g_{V}\right)_{b c}=\exp \left[-\lambda^{a} \varepsilon_{a b c}\right] .
$$

Hence, bosonic configurations that admit unbroken hypersymmetries possess Killing vector-spinors of the form (3.2) provided they are globally well-defined, either for periodic or antiperiodic boundary conditions.

\subsection{Cosmological spacetimes and solutions with conical defects}

Let us focus on an interesting class of circularly symmetric solutions that describe cosmological spacetimes as well as configurations with conical defects. The latter class was introduced in $[10,11]$ while the former one was explored in [12-14]. The thermodynamic properties of cosmological spacetimes have been analyzed in [15-18]. As explained in [9, 19, 20], it is useful to express the solution for a fixed range of the coordinates, so that the Hawking temperature and the chemical potential for the angular momentum manifestly appear in the metric. Hereafter we follow the conventions of [18], and for latter purposes, it is convenient to write the line element in outgoing null coordinates, which reads

$$
d s^{2}=-\frac{4 \pi}{k}\left(\frac{\pi \mathcal{J}^{2}}{k r^{2}}-\mathcal{P}\right) \mu_{\mathcal{P}}^{2} d u^{2}-2 \mu_{\mathcal{P}} d u d r+r^{2}\left[d \phi+\left(\mu_{\mathcal{J}}+\frac{2 \pi \mu_{\mathcal{P}} \mathcal{J}}{k r^{2}}\right) d u\right]^{2} .
$$

Here $\mathcal{P}$ determines the mass, whose associated "chemical potential" relates to the inverse Hawking temperature according to $\mu_{\mathcal{P}}=-\beta^{-1}$. Analogously, $\mu_{\mathcal{J}}$ stands for the chemical potential associated to the angular momentum $\mathcal{J}$. We also assume a non-diagonal form for the Minkowski metric in a local frame, so that its nonvanishing components are given by $\eta_{01}=\eta_{10}=\eta_{22}=1$. The dreibein can then be chosen as

$$
e^{0}=-d r+\frac{2 \pi \mu_{\mathcal{P}} \mathcal{P}}{k} d u+\frac{2 \pi \mathcal{J}}{k}\left(d \phi+\mu_{\mathcal{J}} d u\right), \quad e^{1}=\mu_{\mathcal{P}} d u, \quad e^{2}=r\left(d \phi+\mu_{\mathcal{J}} d u\right),
$$


and hence, the components of the dualized spin connection are given by

$$
\omega^{0}=\frac{2 \pi \mathcal{P}}{k}\left(d \phi+\mu_{\mathcal{J}} d u\right), \quad \omega^{1}=d \phi+\mu_{\mathcal{J}} d u, \quad \omega^{2}=0 .
$$

As explained at the beginning of section 3, since the curvature two-form vanishes, the spin connection (3.6) is locally flat, and it can then be generically written as $\omega=g^{-1} d g$, with

$$
g=\exp \left[\left(J_{1}+\frac{2 \pi \mathcal{P}}{k} J_{0}\right) \hat{\phi}\right]
$$

and $\hat{\phi}=\phi+\mu_{\mathcal{J}} u$.

Note that in the case of $\mathcal{P} \neq 0$, for the spinor and vector representations, the group element $g$ in (3.7) exponentiates as

$$
\begin{aligned}
& g_{S}=\cosh \left[\sqrt{\frac{\pi \mathcal{P}}{k}} \hat{\phi}\right] \mathbb{I}_{2 \times 2}+\sqrt{\frac{k}{\pi \mathcal{P}}} \sinh \left[\sqrt{\frac{\pi \mathcal{P}}{k}} \hat{\phi}\right]\left(J_{1}+\frac{2 \pi \mathcal{P}}{k} J_{0}\right), \\
& g_{V}=\mathbb{I}_{3 \times 3}+\frac{1}{2} \sqrt{\frac{k}{\pi \mathcal{P}}} \sinh \left[2 \sqrt{\frac{\pi \mathcal{P}}{k}} \hat{\phi}\right]\left(J_{1}+\frac{2 \pi \mathcal{P}}{k} J_{0}\right)+\frac{k}{2 \pi \mathcal{P}} \sinh \left[\sqrt{\frac{\pi \mathcal{P}}{k}} \hat{\phi}\right]^{2}\left(J_{1}+\frac{2 \pi \mathcal{P}}{k} J_{0}\right)^{2},
\end{aligned}
$$

respectively, while for $\mathcal{P}=0$, it reduces to

$$
g_{S}=\mathbb{I}_{2 \times 2}+\hat{\phi} J_{1}, \quad g_{V}=\mathbb{I}_{3 \times 3}+\hat{\phi} J_{1}+\frac{1}{2} \hat{\phi}^{2} J_{1}^{2} .
$$

One then concludes that cosmological spacetimes, for which $\mathcal{P}>0$, necessarily break all the hypersymmetries. Indeed, this class of solutions cannot admit globally-defined Killing vector-spinors because, according to (3.8) and (3.9), the (anti)periodic boundary conditions for the vector-spinor $\epsilon_{a}$ in (3.2) fail to be fulfilled.

In the case of configurations with $\mathcal{P}=0$, equations (3.2) and (3.10) imply that the Killing vector-spinor is constant and satisfies:

$$
\frac{3}{2} \Gamma_{1} \epsilon_{a}-\Gamma_{a} \epsilon_{1}=0
$$

so that it fulfills periodic boundary conditions, and possesses a single nonvanishing component given by $\epsilon_{0}^{-}=\eta_{0}^{-}$.

For the remaining case, $\mathcal{P}:=-k j^{2} / \pi<0$, describing solutions with conical defects, the group element in both representations reduces to

$$
\begin{aligned}
& g_{S}=\cos [j \hat{\phi}] \mathbb{I}_{2 \times 2}+\frac{1}{j} \sin [j \hat{\phi}]\left(J_{1}-2 j^{2} J_{0}\right), \\
& g_{V}=\mathbb{I}_{3 \times 3}+\frac{1}{2 j} \sin [2 j \hat{\phi}]\left(J_{1}-2 j^{2} J_{0}\right)+\frac{1}{2 j^{2}} \sin [j \hat{\phi}]^{2}\left(J_{1}-2 j^{2} J_{0}\right)^{2} .
\end{aligned}
$$

Therefore, this class of configurations possesses four independent Killing vector-spinors that fulfill (anti)periodic boundary conditions provided $j$ is a (half-)integer. The explicit form of the Killing vector-spinors is then obtained from (3.2), where $g_{S}$ and $g_{V}$ are given 
by eqs. (3.12) and (3.13). Note that this is the maximum number of hypersymmetries. Indeed, for these configurations the holonomy of the spin connection becomes trivial, which in the spinor representation means that $g_{S}^{-1}(\hat{\phi}) g_{S}(\hat{\phi}+2 \pi)=-\mathbb{I}_{2 \times 2}$, while in the vector representation the condition reads $g_{V}^{-1}(\hat{\phi}) g_{V}(\hat{\phi}+2 \pi)=\mathbb{I}_{3 \times 3}$. It is worth pointing out that if $j$ were different from a (half-)integer, the configurations would not solve the field equations in vacuum. This is because they would possess a conical singularity at the origin, and hence they should necessarily be supported by an external source.

As it occurs in the case of supersymmetry, it is natural to expect that the bosonic global charges fulfill suitable bounds that turn out to be saturated for configurations that possess unbroken hypersymmetries. Indeed, as shown in [21], the bounds that correspond to three-dimensional supergravity with asymptotically flat boundary conditions certainly do so. Actually, the bounds also exclude conical surplus solutions, in particular those whose angular coordinate ranges from zero to $4 \pi j$, with $j>1 / 2$, despite they are maximally supersymmetric. When a negative cosmological constant is considered, this is also the case not only for supergravity [22], but also for hypergravity [9], where in the latter case the bounds turn out to be nonlinear. Thus, one of the main purposes of the following sections is showing how these results can be extended to the case of hypergravity endowed with a suitable set of asymptotically flat boundary conditions, as well as how to recover them in the vanishing cosmological constant limit.

\section{Asymptotically flat behaviour and the hyper-BMS ${ }_{3}$ algebra}

Let us introduce a suitable set of asymptotic conditions that allows to describe the dynamics of asymptotically flat spacetimes in hypergravity. The set must be relaxed enough so as to accommodate the solutions of interest that have been described in section 3.1, and simultaneously, restricted in an appropriate way in order to ensure finiteness of the canonical generators associated to the asymptotic symmetries. In the case of pure General Relativity, a consistent set of boundary conditions indeed exists, whose asymptotic symmetry algebra corresponds to $\mathrm{BMS}_{3}$ with a nontrivial central extension [23-25]. These results have been extended to the case of supergravity [21], as well as for General Relativity coupled to higher spin fields $[17,18,26,27]$. In order to carry out this task in hypergravity, we take advantage of the Chern-Simons formulation of the theory, depicted in section 2. Since the hypersymmetry generators are $\Gamma$-traceless, it is useful to get rid of $Q_{2}=Q_{1} \Gamma_{0}-Q_{0} \Gamma_{1}$, so that once the remaining generators are relabeled according to

$$
\begin{array}{lll}
\hat{\mathcal{J}}_{-1}=-2 J_{0}, & \hat{\mathcal{J}}_{1}=J_{1}, & \hat{\mathcal{J}}_{0}=J_{2}, \\
\hat{\mathcal{P}}_{-1}=-2 P_{0}, & \hat{\mathcal{P}}_{1}=P_{1}, & \hat{\mathcal{P}}_{0}=P_{2}, \\
\hat{\mathcal{Q}}_{-\frac{3}{2}}=2^{\frac{5}{4}} \sqrt{3} Q_{+0}, & \hat{\mathcal{Q}}_{-\frac{1}{2}}=2^{\frac{3}{4}} \sqrt{3} Q_{-0}, \\
\hat{\mathcal{Q}}_{\frac{1}{2}}=-2^{\frac{1}{4} \sqrt{3} Q_{+1},} & \hat{\mathcal{Q}}_{\frac{3}{2}}=-2^{-\frac{1}{4}} \sqrt{3} Q_{-1},
\end{array}
$$


the hyper-Poincaré algebra (2.2) reads

$$
\begin{aligned}
& {\left[\hat{\mathcal{J}}_{m}, \hat{\mathcal{J}}_{n}\right]=(m-n) \hat{\mathcal{J}}_{m+n}} \\
& {\left[\hat{\mathcal{J}}_{m}, \hat{\mathcal{P}}_{n}\right]=(m-n) \hat{\mathcal{P}}_{m+n}} \\
& {\left[\hat{\mathcal{J}}_{m}, \hat{\mathcal{Q}}_{p}\right]=\left(\frac{3 m}{2}-p\right) \hat{\mathcal{Q}}_{m+p},} \\
& \left\{\hat{\mathcal{Q}}_{p}, \hat{\mathcal{Q}}_{q}\right\}=\frac{1}{4}\left(6 p^{2}-8 p q+6 q^{2}-9\right) \hat{\mathcal{P}}_{p+q},
\end{aligned}
$$

with $m, n= \pm 1,0$, and $p, q= \pm \frac{1}{2}, \pm \frac{3}{2}$.

Thus, following the lines of [28], and as explained in [21,27], the radial dependence of the asymptotic form of the gauge field can be gauged away by a suitable group element of the form $h=e^{\frac{r}{2} \hat{\mathcal{P}}_{-1}}$, so that

$$
A=h^{-1} a h+h^{-1} d h,
$$

and hence, the remaining analysis can be entirely performed in terms of the connection $a=a_{u} d u+a_{\phi} d \phi$, that depends only on time and the angular coordinate. As explained in $[19,20]$, one starts prescribing the asymptotic form of the dynamical gauge field at a fixed time slice with $u=u_{0}$, so that the asymptotic fall-off of $a_{\phi}$ is assumed to be such that the deviations with respect to the reference background go along the highest weight generators of (4.2). Choosing the reference background to be given by the null orbifold [29], that corresponds to the configuration in (3.4) with $\mathcal{J}=P=0$, the asymptotic form of the dynamical field reads

$$
a_{\phi}=\hat{\mathcal{J}}_{1}-\frac{\pi}{k}\left(\mathcal{J} \hat{\mathcal{P}}_{-1}+\mathcal{P} \hat{\mathcal{J}}_{-1}-\frac{\psi}{3} \hat{\mathcal{Q}}_{-\frac{3}{2}}\right)
$$

where $\mathcal{J}, \mathcal{P}$ and $\psi$ stand for arbitrary functions of $u, \phi$. The asymptotic symmetries then correspond to gauge transformations $\delta a=d \lambda+[a, \lambda]$ that preserve the form of (4.4). Therefore, the hyper-Poincaré-valued parameter $\lambda$ is found to depend on three arbitrary functions of $u$ and $\phi$, so that

$$
\lambda=T \hat{\mathcal{P}}_{1}+Y \hat{\mathcal{J}}_{1}+\mathcal{E} \hat{\mathcal{Q}}_{\frac{3}{2}}+\eta_{\left(\frac{3}{2}\right)}[T, Y, \mathcal{E}]
$$

where $\mathcal{E}$ is Grassmann-valued, and

$$
\begin{aligned}
\eta_{\left(\frac{3}{2}\right)}[T, Y, \mathcal{E}]= & -T^{\prime} \hat{\mathcal{P}}_{0}-Y^{\prime} \hat{\mathcal{J}}_{0}-\mathcal{E}^{\prime} \hat{\mathcal{Q}}_{\frac{1}{2}}-\frac{1}{2}\left(\frac{2 \pi}{k} Y \mathcal{P}-Y^{\prime \prime}\right) \hat{\mathcal{J}}_{-1} \\
& -\frac{\pi}{k}\left(T \mathcal{P}+Y \mathcal{J}-\frac{3}{2} i \psi \mathcal{E}-\frac{k}{2 \pi} T^{\prime \prime}\right) \hat{\mathcal{P}}_{-1}-\frac{1}{2}\left(\frac{3 \pi}{k} \mathcal{E} \mathcal{P}-\mathcal{E}^{\prime \prime}\right) \hat{\mathcal{Q}}_{-\frac{1}{2}} \\
& -\frac{\pi}{3 k}\left(Y \psi-\frac{7}{2} \mathcal{E}^{\prime} \mathcal{P}-\frac{3}{2} \mathcal{E} \mathcal{P}^{\prime}+\frac{k}{2 \pi} \mathcal{E}^{\prime \prime \prime}\right) \hat{\mathcal{Q}}_{-\frac{3}{2}} ;
\end{aligned}
$$

while the transformation law of the fields reads

$$
\begin{aligned}
& \delta \mathcal{P}=2 \mathcal{P} Y^{\prime}+\mathcal{P}^{\prime} Y-\frac{k}{2 \pi} Y^{\prime \prime \prime} \\
& \delta \mathcal{J}=2 \mathcal{J} Y^{\prime}+\mathcal{J}^{\prime} Y+2 \mathcal{P} T^{\prime}+\mathcal{P}^{\prime} T-\frac{k}{2 \pi} T^{\prime \prime \prime}+\frac{5}{2} i \psi \mathcal{E}^{\prime}+\frac{3}{2} i \psi^{\prime} \mathcal{E} \\
& \delta \psi=\frac{5}{2} \psi Y^{\prime}+\psi^{\prime} Y-\frac{9 \pi}{2 k} \mathcal{P}^{2} \mathcal{E}+\frac{3}{2} \mathcal{P}^{\prime \prime} \mathcal{E}+5 \mathcal{P}^{\prime} \mathcal{E}^{\prime}+5 \mathcal{P} \mathcal{E}^{\prime \prime}-\frac{k}{2 \pi} \mathcal{E}^{\prime \prime \prime \prime} .
\end{aligned}
$$


Hereafter, prime stands for $\partial_{\phi}$. Since the time evolution of $a_{\phi}$ corresponds to a gauge transformation parametrized by the Lagrange multiplier $a_{u}$, its asymptotic form will be maintained along different time slices provided $a_{u}$ is of the allowed form, i. e.,

$$
a_{u}=\lambda\left[\mu_{\mathcal{P}}, \mu_{\mathcal{J}}, \mu_{\psi}\right]
$$

where the chemical potentials $\mu_{\mathcal{P}}, \mu_{\mathcal{J}}, \mu_{\psi}$ stand for arbitrary functions of $u$, $\phi$, that are assumed to be fixed at the boundary. Consistency then demands that the field equations, which now reduce to

$$
\begin{aligned}
& \dot{\mathcal{P}}=2 \mathcal{P} \mu_{\mathcal{J}}{ }^{\prime}+\mathcal{P}^{\prime} \mu_{\mathcal{J}}-\frac{k}{2 \pi} \mu_{\mathcal{J}}{ }^{\prime \prime \prime}, \\
& \dot{\mathcal{J}}=2 \mathcal{J} \mu_{\mathcal{J}}{ }^{\prime}+\mathcal{J}^{\prime} \mu_{\mathcal{J}}+2 \mathcal{P} \mu_{\mathcal{P}}{ }^{\prime}+\mathcal{P}^{\prime} \mu_{\mathcal{P}}-\frac{k}{2 \pi} \mu_{\mathcal{P}}^{\prime \prime \prime}+\frac{5}{2} i \psi \mu_{\psi}{ }^{\prime}+\frac{3}{2} i \psi^{\prime} \mu_{\psi}, \\
& \dot{\psi}=\frac{5}{2} \psi \mu_{\mathcal{J}}{ }^{\prime}+\psi^{\prime} \mu_{\mathcal{J}}-\frac{9 \pi}{2 k} \mathcal{P}^{2} \mu_{\psi}+\frac{3}{2} \mathcal{P}^{\prime \prime} \mu_{\psi}+5 \mathcal{P}^{\prime} \mu_{\psi}{ }^{\prime}+5 \mathcal{P} \mu_{\psi}{ }^{\prime \prime}-\frac{k}{2 \pi} \mu_{\psi}{ }^{\prime \prime \prime \prime},
\end{aligned}
$$

have to hold in the asymptotic region, while the parameters of the asymptotic symmetries fulfill the following conditions

$$
\begin{aligned}
\dot{Y} & =\mu_{\mathcal{J}} Y^{\prime}-\mu_{\mathcal{J}}{ }^{\prime} Y, \\
\dot{T} & =\mu_{\mathcal{J}} T^{\prime}-\mu_{\mathcal{J}}{ }^{\prime} T+\mu_{\mathcal{P}} Y^{\prime}-\mu_{\mathcal{P}}{ }^{\prime} Y+\frac{9 \pi}{k} i \mu_{\psi} \mathcal{E} \mathcal{P}-\frac{3}{2} i \mu_{\psi}{ }^{\prime \prime} \mathcal{E}+2 i \mu_{\psi}{ }^{\prime} \mathcal{E}^{\prime}-\frac{3}{2} i \mu_{\psi} \mathcal{E}^{\prime \prime}, \\
\dot{\mathcal{E}} & =\frac{3}{2} \mu_{\psi} Y^{\prime}-\mu_{\psi}{ }^{\prime} Y-\frac{3}{2} \mu_{\mathcal{J}} \mathcal{E}+\mu_{\mathcal{J}} \mathcal{E}^{\prime}
\end{aligned}
$$

which are needed in order to ensure that the global charges are conserved. ${ }^{1}$

Following the Regge-Teitelboim approach [30], the variation of the canonical generators is found to be generically given by

$$
\delta Q[\lambda]=-\frac{k}{2 \pi} \int\left\langle\lambda \delta a_{\phi}\right\rangle d \phi
$$

which by virtue of (4.4) and (4.5), up to an arbitrary constant without variation, integrate as

$$
Q[T, Y, \mathcal{E}]=-\int(T \mathcal{P}+Y \mathcal{J}-i \mathcal{E} \psi) d \phi
$$

It is worth highlighting that the global charges are manifestly independent of the radial coordinate $r$. Therefore, the boundary can be located at an arbitrary fixed value $r=r_{0}$, and it corresponds to a timelike surface with the topology of a cylinder.

Since the Poisson brackets fulfill $\left\{Q\left[\lambda_{1}\right], Q\left[\lambda_{2}\right]\right\}=\delta_{\lambda_{2}} Q\left[\lambda_{1}\right]$, the algebra of the canonical generators can be directly obtained from the transformation law of the fields in (4.7).

\footnotetext{
${ }^{1}$ Since global symmetries are necessarily contained within the asymptotic ones, these results provide an interesting alternative path to find the explicit expression of the Killing vector-spinors. See appendix B.
} 
Expanding in Fourier modes, $X=\frac{1}{2 \pi} \sum_{n} X_{n} e^{i n \phi}$, the nonvanishing Poisson brackets read

$$
\begin{aligned}
i\left\{\mathcal{J}_{m}, \mathcal{J}_{n}\right\} & =(m-n) \mathcal{J}_{m+n}, \\
i\left\{\mathcal{J}_{m}, \mathcal{P}_{n}\right\} & =(m-n) \mathcal{P}_{m+n}+k m^{3} \delta_{m+n, 0}, \\
i\left\{\mathcal{J}_{m}, \psi_{n}\right\} & =\left(\frac{3 m}{2}-n\right) \psi_{m+n}, \\
i\left\{\psi_{m}, \psi_{n}\right\} & =\frac{1}{2}\left(3 m^{2}-4 m n+3 n^{2}\right) \mathcal{P}_{m+n}+\frac{9}{4 k} \sum_{q} \mathcal{P}_{m+n-q} \mathcal{P}_{q}+k m^{4} \delta_{m+n, 0},
\end{aligned}
$$

where the modes of the generators $\psi_{m}$ are labeled by (half-)integers when the fermions fulfill (anti)periodic boundary conditions.

It is then clear that, with respect to $\mathcal{J}_{m}$, the conformal weight of the generators $\mathcal{P}_{m}$ and $\psi_{n}$, is given by 2 and $5 / 2$, respectively. Note that the subset spanned by $\mathcal{J}_{m}$ and $\mathcal{P}_{m}$ corresponds to the $\mathrm{BMS}_{3}$ algebra of General Relativity with the same central extension, and hence (4.13) stands for its hypersymmetric extension that is manifestly nonlinear.

It is useful to perform the following shift in the generators:

$$
\mathcal{P}_{n} \rightarrow \mathcal{P}_{n}-\frac{k}{2} \delta_{n, 0},
$$

so that the algebra now reads

$$
\begin{aligned}
i\left\{\mathcal{J}_{m}, \mathcal{J}_{n}\right\}= & (m-n) \mathcal{J}_{m+n}, \\
i\left\{\mathcal{J}_{m}, \mathcal{P}_{n}\right\}= & (m-n) \mathcal{P}_{m+n}+k m\left(m^{2}-1\right) \delta_{m+n, 0}, \\
i\left\{\mathcal{J}_{m}, \psi_{n}\right\}= & \left(\frac{3 m}{2}-n\right) \psi_{m+n}, \\
i\left\{\psi_{m}, \psi_{n}\right\}= & \frac{1}{4}\left(6 m^{2}-8 m n+6 n^{2}-9\right) \mathcal{P}_{m+n}+\frac{9}{4 k} \sum_{q} \mathcal{P}_{m+n-q} \mathcal{P}_{q} \\
& +k\left(m^{2}-\frac{9}{4}\right)\left(m^{2}-\frac{1}{4}\right) \delta_{m+n, 0},
\end{aligned}
$$

in agreement with the result that has been recently anticipated in [6]. Indeed, dropping the nonlinear terms in (4.15), when the fermions fulfill antiperiodic boundary conditions, the wedge algebra, which is spanned by the subset of $\left\{\mathcal{J}_{m}, \mathcal{P}_{m}, \psi_{n}\right\}$ with $m= \pm 1,0$ and $n= \pm 3 / 2, \pm 1 / 2$, reduces to the hyper-Poincaré algebra in eq. (4.2).

It can also be seen that the hyper-BMS 3 algebra (4.13) turns out to be a subset of a precise Inönü-Wigner contraction of the direct sum of the $\mathrm{W}_{(2,4)}$ algebra with its hypersymmetric extension $\mathrm{W}_{\left(2, \frac{5}{2}, 4\right)}$. This is the main subject of the next subsection.

\subsection{Flat limit of the asymptotic symmetry algebra from the case of negative cosmological constant}

It has been recently shown that the asymptotic symmetries of three-dimensional hypergravity with negative cosmological constant are spanned by two copies of the classical limit of the $\mathrm{WB}_{2}$ algebra [9]. This algebra is also known as $\mathrm{W}_{\left(2, \frac{5}{2}, 4\right)}$ and corresponds to the hypersymmetric extension of $\mathrm{W}_{(2,4)}[31,32]$. The hypergravity theory that was discussed in [9] 
possesses the minimum number of hypersymmetries in each sector, so that the gauge group is given by $O S p(1 \mid 4) \otimes O S p(1 \mid 4)$. In analogy with the case of three-dimensional supergravity [33], one may say that the theory aforementioned corresponds to the $\mathcal{N}=(1,1) \mathrm{AdS}_{3}$ hypergravity. In this sense, there are two inequivalent minimal locally hypersymmetric extensions of General Relativity with negative cosmological constant, which correspond to the $(1,0)$ and the $(0,1)$ theories. It is then simple to verify that both minimal theories possess the same vanishing cosmological constant limit, and hence in order to proceed with the analysis we will consider the $(0,1)$ one, whose gauge group is given by $S p(4) \otimes O S p(1 \mid 4)$. According to [9], the asymptotic symmetry algebra of the minimal hypergravity theory with negative cosmological constant then corresponds to $\mathrm{W}_{(2,4)} \oplus \mathrm{W}_{\left(2, \frac{5}{2}, 4\right)}$.

The classical limit of the $\mathrm{W}_{\left(2, \frac{5}{2}, 4\right)}$ algebra reads

$$
\begin{aligned}
i\left\{\mathcal{L}_{m}, \mathcal{L}_{n}\right\}= & (m-n) \mathcal{L}_{m+n}+\frac{\kappa}{2} m^{3} \delta_{m+n, 0} \\
i\left\{\mathcal{L}_{m}, \mathcal{U}_{n}\right\}= & (3 m-n) \mathcal{U}_{m+n}, \\
i\left\{\mathcal{L}_{m}, \Psi_{n}\right\}= & \left(\frac{3 m}{2}-n\right) \Psi_{m+n} \\
i\left\{\mathcal{U}_{m}, \mathcal{U}_{n}\right\}= & \frac{1}{2^{2} 3^{2}}(m-n)\left(3 m^{4}-2 m^{3} n+4 m^{2} n^{2}-2 m n^{3}+3 n^{4}\right) \mathcal{L}_{m+n} \\
& +\frac{1}{6}(m-n)\left(m^{2}-m n+n^{2}\right) \mathcal{U}_{m+n}-\frac{2^{3} 3 \pi}{\kappa}(m-n) \Lambda_{m+n}^{(6)} \\
& -\frac{7^{2} \pi}{3^{2} \kappa}(m-n)\left(m^{2}+4 m n+n^{2}\right) \Lambda_{m+n}^{(4)}+\frac{\kappa}{2^{3} 3^{2}} m^{7} \delta_{m+n, 0}, \\
i\left\{\mathcal{U}_{m}, \Psi_{n}\right\}= & \frac{1}{2^{2} 3}\left(m^{3}-4 m^{2} n+10 m n^{2}-20 n^{3}\right) \Psi_{m+n}-\frac{23 \pi}{3 \kappa} i \Lambda_{m+n}^{(11 / 2)} \\
& +\frac{\pi}{3 \kappa}(23 m-82 n) \Lambda_{m+n}^{(9 / 2)}, \\
i\left\{\Psi_{m}, \Psi_{n}\right\}= & \mathcal{U}_{m+n}+\frac{1}{2}\left(m^{2}-\frac{4}{3} m n+n^{2}\right) \mathcal{L}_{m+n}+\frac{3 \pi}{\kappa} \Lambda_{m+n}^{(4)}+\frac{\kappa}{6} m^{4} \delta_{m+n, 0},
\end{aligned}
$$

where the fermionic modes are labeled by (half-)integers in the case of (anti)periodic boundary conditions, and $\Lambda_{m}^{(l)}=\int \Lambda^{(l)} e^{-i m \phi} d \phi$ stand for the mode expansion of the nonlinear terms, given by

$$
\begin{aligned}
\Lambda^{(4)} & =\mathcal{L}^{2} \\
\Lambda^{(9 / 2)} & =\mathcal{L} \Psi \\
\Lambda^{(11 / 2)} & =\frac{27}{23} \mathcal{L}^{\prime} \Psi \\
\Lambda^{(6)} & =-\frac{7}{18} \mathcal{U} \mathcal{L}-\frac{8 \pi}{3 \kappa} \mathcal{L}^{3}+\frac{295}{432}\left(\mathcal{L}^{\prime}\right)^{2}+\frac{22}{27} \mathcal{L}^{\prime \prime} \mathcal{L}+\frac{25}{12} i \Psi \Psi^{\prime} .
\end{aligned}
$$

The bosonic generators $\mathcal{L}_{m}$ and $\mathcal{U}_{m}$ span the $\mathrm{W}_{(2,4)}$ subalgebra.

In order to take the vanishing cosmological constant limit of the asymptotic symmetry algebra of the minimal theory, given by $\mathrm{W}_{(2,4)} \oplus \mathrm{W}_{\left(2, \frac{5}{2}, 4\right)}$, it is useful to perform the following 
change of basis:

$$
\begin{array}{rlrl}
\mathcal{P}_{n} & =\frac{1}{\ell}\left(\mathcal{L}_{n}^{+}+\mathcal{L}_{-n}^{-}\right), & \mathcal{J}_{n} & =\mathcal{L}_{n}^{+}-\mathcal{L}_{-n}^{-}, \\
\mathcal{W}_{n}=\frac{1}{\sqrt{\ell}}\left(\mathcal{U}_{n}^{+}+\mathcal{U}_{-n}^{-}\right), & \mathcal{V}_{n}=\frac{1}{\sqrt{\ell}}\left(\mathcal{U}_{n}^{+}-\mathcal{U}_{-n}^{-}\right), & \psi_{n}=\sqrt{\frac{6}{\ell}} \Psi_{n}^{+},
\end{array}
$$

where $\mathcal{L}_{n}^{-}, \mathcal{U}_{n}^{-}$stand for the generators of the (left) $\mathrm{W}_{(2,4)}$ algebra, and $\mathcal{L}_{n}^{+}, \mathcal{U}_{n}^{+}, \psi_{n}^{+}$span the (right) $\mathrm{W}_{\left(2, \frac{5}{2}, 4\right)}$ algebra. Therefore, rescaling the level according to $\kappa=k \ell$, in the large AdS radius limit, $\ell \rightarrow \infty$, one obtains that the nonvanishing brackets of the contracted algebra read

$$
\begin{aligned}
i\left\{\mathcal{J}_{m}, \mathcal{J}_{n}\right\}= & (m-n) \mathcal{J}_{m+n} \\
i\left\{\mathcal{J}_{m}, \mathcal{P}_{n}\right\}= & (m-n) \mathcal{P}_{m+n}+k m^{3} \delta_{m+n, 0} \\
i\left\{\mathcal{J}_{m}, \mathcal{W}_{n}\right\}= & (3 m-n) \mathcal{W}_{m+n} \\
i\left\{\mathcal{J}_{m}, \mathcal{V}_{n}\right\}= & (3 m-n) \mathcal{V}_{m+n}, \\
i\left\{\mathcal{V}_{m}, \mathcal{W}_{n}\right\}= & \frac{1}{2^{2} 3^{2}}(m-n)\left(3 m^{4}-2 m^{3} n+4 m^{2} n^{2}-2 m n^{3}+3 n^{4}\right) \mathcal{P}_{m+n} \\
& -\frac{2^{3} \pi}{k}(m-n) \tilde{\Lambda}_{m+n}^{(6)}-\frac{7^{2}}{3^{2} 4 k}(m-n)\left(m^{2}+4 m n+n^{2}\right) \sum_{q} \mathcal{P}_{m+n-q} \mathcal{P}_{q} \\
& +\frac{k}{2^{2} 3^{2}} m^{7} \delta_{m+n, 0}, \\
i\left\{\mathcal{J}_{m}, \psi_{n}\right\}= & \left(\frac{3 m}{2}-n\right) \psi_{m+n}, \\
i\left\{\psi_{m}, \psi_{n}\right\}= & \frac{1}{2}\left(3 m^{2}-4 m n+3 n^{2}\right) \mathcal{P}_{m+n}+\frac{9}{4 k} \sum_{q} \mathcal{P}_{m+n-q} \mathcal{P}_{q}+k m^{4} \delta_{m+n, 0}
\end{aligned}
$$

with

$$
\tilde{\Lambda}^{(6)}=-\frac{7}{12} \mathcal{W} \mathcal{P}-\frac{2 \pi}{k} \mathcal{P}^{3}+\frac{295}{288}\left(\mathcal{P}^{\prime}\right)^{2}+\frac{11}{9} \mathcal{P} \mathcal{P}^{\prime \prime} .
$$

It is then apparent that one can consistently get rid of the (conformal) spin-4 generators $\mathcal{V}_{m}, \mathcal{W}_{n}$, since the Inönü-Wigner contraction of $\mathrm{W}_{(2,4)} \oplus \mathrm{W}_{\left(2, \frac{5}{2}, 4\right)}$ in eq. (4.22) possesses a subset spanned by $\left\{\mathcal{P}_{m}, \mathcal{J}_{m}, \psi_{m}\right\}$, which precisely corresponds to the hyper-BMS 3 algebra in (4.13). Note that this is just a reflection of the fact that in the vanishing cosmological constant limit, the hypergravity theory can be consistently formulated without the need of spin-4 fields.

\section{Hypersymmetry bounds}

In the case of hypergravity with negative cosmological constant, it has been recently shown that the anticommutator of the generators of the asymptotic hypersymmetries implies the existence of interesting nonlinear bounds for the bosonic charges, that saturate for configurations that admit unbroken hypersymmetries [9]. In this section, following these lines, we explicitly show that this is also the case for hypergravity with asymptotically flat 
boundary conditions. In order to perform this task, it is useful to assume that the bosonic global charges are just determined by the zero modes. Indeed, as explained in [20], a generic bosonic configuration can be brought to the "rest frame" through the action of suitable elements of the asymptotic symmetry algebra. The searched for bounds can then be found along the same semi-classical reasoning as in the case of supergravity [22, 34-39]. Hence, the fermionic bracket in (4.13) becomes an anticommutator, which in the rest frame, and for $m=-n=p$, reads

$$
\frac{1}{2 \pi}\left(\hat{\psi}_{p} \hat{\psi}_{-p}+\hat{\psi}_{-p} \hat{\psi}_{p}\right)=5 p^{2} \hat{\mathcal{P}}+\frac{9 \pi}{2 k} \hat{\mathcal{P}}^{2}+\frac{k}{2 \pi} p^{4} \geq 0,
$$

with $\hat{\mathcal{P}}_{0}=2 \pi \hat{\mathcal{P}}$. Thus, since the left-hand side of $(5.1)$ is a positive-definite hermitian operator, in the classical limit, and for any value of the (half-)integer $p$, the energy has to fulfill the following bounds:

$$
\left(p^{2}+\frac{9 \pi}{k} \mathcal{P}\right)\left(p^{2}+\frac{\pi}{k} \mathcal{P}\right) \geq 0
$$

which are manifestly nonlinear.

Note that for any configuration with $\mathcal{P}>0$, the bounds in (5.2) are automatically fulfilled, but never saturate. Indeed, this is the case of the cosmological spacetimes in (3.4), which goes by hand with the fact that they do not admit globally-defined Killing vectorspinors, and hence, break all the hypersymmetries.

These bounds are also clearly fulfilled in the case of $\mathcal{P}=0$, and for fermions with periodic boundary conditions, the one for $p=0$ is saturated. This relates to the fact that this class of configurations, that includes the null orbifold, possesses a single unbroken hypersymmetry spanned by a constant Killing vector-spinor.

In the case of $\mathcal{P}<0$, the class of smooth configurations are the ones for which the holonomy of the connection around an angular cycle is trivial. This means that they are maximally (hyper)symmetric, and then possess four Killing vector-spinors. As explained in section 3.1 , their energy is given by $\mathcal{P}=-k j^{2} / \pi$, and the bounds in (5.2) then reduce to

$$
\left(p^{2}-9 j^{2}\right)\left(p^{2}-j^{2}\right) \geq 0 .
$$

Remarkably, the bounds are only fulfilled in the case of $j^{2}=1 / 4$, so that four of them saturate, corresponding to $p= \pm 1 / 2$, and $p= \pm 3 / 2$. This is the case of Minkowski spacetime $(\mathcal{P}=-k / 4 \pi)$, with four independent Killing vector-spinors that fulfill antiperiodic boundary conditions. Hence, in spite of being maximally hypersymmetric, smooth solutions whose energy is lower than the one of Minkowski spacetime are excluded by the hypersymmetry bounds.

It is worth highlighting that one arrives to similar conclusions in the case of asymptotically flat spacetimes in supergravity [21]. In fact, despite the analysis is fairly different, the supersymmetry bounds precisely select the same spectrum, including the corresponding ground states who saturate the bounds for spinors that fulfill different periodicity conditions. 


\section{Hypergravity reloaded}

Let us look for a different theory of three-dimensional (hyper)gravity that is still compatible with the asymptotically flat boundary conditions described above, but now allowing the presence of spacetime torsion even in vacuum. For simplicity, we consider modifications such that the field equations are still of first order for the dreibein and the spin connection. One interesting possibility is to include additional terms, so that the action is given by

$$
I_{\gamma}=\frac{k}{4 \pi} \int 2 R^{a} e_{a}+\gamma^{2} \epsilon_{a b c} e^{a} e^{b} e^{c}+2 \gamma T^{a} e_{a} .
$$

Remarkably, despite the second (volume) term in the action looks like a cosmological constant $\Lambda=-3 \gamma^{2}$, the field equations actually imply that the Riemann curvature vanishes. Indeed, the presence of the last (parity-odd) term in the action has the effect of making the volume term to act as the source for a fully antisymmetric torsion in vacuum, being proportional to the volume element, given by

$$
T^{a}=-\gamma \varepsilon^{a b c} e_{b} e_{c}
$$

so that the remaining field equations fix the curvature two-form according to

$$
R^{a}=\frac{1}{2} \gamma^{2} \varepsilon^{a b c} e_{b} e_{c}
$$

Therefore, eq. (6.2) implies that the spin connection splits as $\omega^{a}=\bar{\omega}^{a}+\kappa^{a}$, where $\bar{\omega}^{a}$ is the (torsionless) Levi-Civita connection, and the contorsion reads $\kappa^{a}=-\gamma e^{a}$. The curvature two-form is then given by $R^{a}=\bar{R}^{a}+\frac{1}{2} \gamma^{2} \varepsilon^{a b c} e_{b} e_{c}$, and hence, equation (6.3) implies the vanishing of the Riemann tensor, i. e., $\bar{R}_{a}=\frac{1}{4} \varepsilon_{a \rho \tau} R^{\rho \tau}{ }_{\mu \nu} d x^{\mu} d x^{\nu}=0$.

The most general theory that possesses the features described above is obtained by considering the addition of the Lorentz-Chern-Simons form, $L(\omega)=\omega^{a} d \omega_{a}+\frac{1}{3} \varepsilon_{a b c} \omega^{a} \omega^{b} \omega^{c}$, with an independent coupling $\mu$, provided the remaining couplings in (6.1) are suitable shifted. The action is then given by

$$
I_{\mu, \gamma}=\frac{k}{4 \pi} \int 2(1+\mu \gamma) R^{a} e_{a}+\gamma^{2}\left(1+\mu \frac{\gamma}{3}\right) \epsilon_{a b c} e^{a} e^{b} e^{c}+\mu L(\omega)+\gamma(2+\mu \gamma) T^{a} e_{a} .
$$

Noteworthy, despite the fact that the Lorentz-Chern-Simons form is not a boundary term, the shifts in the other couplings are such that the field equations in vacuum just become reshuffled, coinciding with the previous ones for $\mu=0$, given by (6.2) and (6.3). Actually, one should highlight that both actions, (6.1) and (6.4), differ off-shell, which reflects through the fact that the canonical generators do not have the same form. Consequently, as in the case of supergravity [21], the asymptotic symmetry algebra of the latter acquires an additional central extension with respect to the former (see below).

The locally hypersymmetric extension of the theory described by (6.4) is given by the following action

$$
I_{\mu, \gamma, \psi}=I_{\mu, \gamma}+\frac{k}{4 \pi} \int i \bar{\psi}_{a}\left(D+\frac{3}{2} \gamma e^{b} \Gamma_{b}\right) \psi^{a},
$$


which is invariant under the following local hypersymmetry transformations:

$$
\delta e^{a}=\frac{3}{2} i \bar{\epsilon}_{b} \Gamma^{a} \psi^{b}, \quad \delta \omega^{a}=-\frac{3}{2} i \gamma \bar{\epsilon}_{b} \Gamma^{a} \psi^{b}, \quad \delta \psi^{a}=D \epsilon^{a}+\frac{3}{2} \gamma e^{b} \Gamma_{b} \epsilon^{a}-\gamma e_{b} \Gamma^{a} \epsilon^{b} .
$$

The field equations now read

$$
\begin{aligned}
R^{a} & =\frac{1}{2} \gamma^{2} \varepsilon^{a b c} e_{b} e_{c}-\frac{3}{4} i \gamma \bar{\psi}_{b} \Gamma^{a} \psi^{b}, \quad T^{a}=-\gamma \varepsilon^{a b c} e_{b} e_{c}+\frac{3}{4} i \bar{\psi}_{b} \Gamma^{a} \psi^{b}, \\
D \psi^{a} & =-\frac{3}{2} \gamma e^{b} \Gamma_{b} \psi^{a}+\gamma e_{b} \Gamma^{a} \psi^{b} .
\end{aligned}
$$

Note that in the case of $\mu=\gamma=0$, the action (6.5), the transformations rules (6.6), and the field equations (6.7), reduce to the ones of the locally hypersymmetric extension of General Relativity, given by eqs. (2.5), (2.8), and (2.7), respectively.

As outlined in [6], in analogy with the case of supergravity [21, 40], the action (6.4) can be formulated as a Chern-Simons one for the hyper-Poincaré group (2.2) by virtue of a simple modification of the invariant bilinear form, and a suitable shift of the spin connection. Indeed, the invariant bilinear form in (2.3) can be consistently modified to admit an additional nonvanishing component given by

$$
\left\langle J_{a}, J_{b}\right\rangle=\mu \eta_{a b},
$$

so that the Chern-Simons action (2.4) now depends on a different hyper-Poincaré-algebravalued gauge field, defined as

$$
A=e^{a} P_{a}+\hat{\omega}^{a} J_{a}+\psi_{a}^{\alpha} Q_{\alpha}^{a},
$$

with $\hat{\omega}^{a}:=\omega^{a}+\gamma e^{a}$. Therefore, in terms of the covariant derivative with respect to $\hat{\omega}^{a}$ and its corresponding curvature, given by $\hat{D}$ and $\hat{R}^{a}$, respectively, up to a surface term, the Chern-Simons action reduces to

$$
I_{\mu, \gamma, \psi}=\frac{k}{4 \pi} \int 2 \hat{R}^{a} e_{a}+\mu L(\hat{\omega})+i \bar{\psi}_{a} \hat{D} \psi^{a},
$$

which precisely agrees with (6.5). Note that the field equations (6.7) correspond to the vanishing of the components of the curvature associated to (6.9), so that they can be compactly written as $F=d A+A^{2}=0$, being manifestly covariant under the full hyperPoincaré group.

One of the advantages of having formulated the extension of hypergravity with parityodd terms as a Chern-Simons theory, is that its asymptotically flat structure can be directly obtained along the lines of the results in section 4 .

The asymptotically flat boundary conditions for the connection (6.9) are then proposed to be precisely as in eqs. (4.3), (4.4), and (4.8), so that the asymptotic fall-off of the spin connection $\omega^{a}$ becomes modified. Therefore, the asymptotic symmetries remain the same as in section 4 , being spanned by the hyper-Poincaré algebra valued parameter $\lambda=\lambda[T, Y, \mathcal{E}]$ given by (4.5). The global charges are then found to acquire a correction due to the additional component of the invariant bilinear form in (6.8), so that they now read

$$
Q[T, Y, \mathcal{E}]=-\int(T \mathcal{P}+Y \tilde{\mathcal{J}}-i \mathcal{E} \psi) d \phi,
$$


with $\tilde{\mathcal{J}}=\mathcal{J}+\mu \mathcal{P}$, and do not depend on the parameter $\gamma$. Note that the shift in the canonical generator associated to $Y$ implies that in the extended theory, even static configurations, as it is the case of Minkowski spacetime, may carry angular momentum.

It is then simple to verify that, once the canonical generators are expanded in modes, their nonvanishing Poisson brackets are given by

$$
\begin{aligned}
i\left\{\tilde{\mathcal{J}}_{m}, \tilde{\mathcal{J}}_{n}\right\} & =(m-n) \tilde{\mathcal{J}}_{m+n}+\mu k m^{3} \delta_{m+n, 0} \\
i\left\{\tilde{\mathcal{J}}_{m}, \mathcal{P}_{n}\right\} & =(m-n) \mathcal{P}_{m+n}+k m^{3} \delta_{m+n, 0}, \\
i\left\{\tilde{\mathcal{J}}_{m}, \psi_{n}\right\} & =\left(\frac{3 m}{2}-n\right) \psi_{m+n}, \\
i\left\{\psi_{m}, \psi_{n}\right\} & =\frac{1}{2}\left(3 m^{2}-4 m n+3 n^{2}\right) \mathcal{P}_{m+n}+\frac{9}{4 k} \sum_{q} \mathcal{P}_{m+n-q} \mathcal{P}_{q}+k m^{4} \delta_{m+n, 0},
\end{aligned}
$$

which corresponds to a hypersymmetric extension of the $\mathrm{BMS}_{3}$ algebra, with an additional central extension along its Virasoro subalgebra.

\section{General Relativity minimally coupled to half-integer spin fields}

In the generic case of fermionic fields of spin $n+\frac{3}{2}$, and in the absence of cosmological constant, the hypergravity action reads $[5,6]$

$$
I=\frac{k}{4 \pi} \int 2 R^{a} e_{a}+i \bar{\psi}_{a_{1} \ldots a_{n}} D \psi^{a_{1} \ldots a_{n}}
$$

where $\psi_{a_{1} \ldots a_{n}}$ describes a Grassmann-valued 1-form that is $\Gamma$-traceless, i. e., $\Gamma^{a_{1}} \psi_{a_{1} \ldots a_{n}}=$ 0 , and completely symmetric in its vector indices. Its covariant derivative can be conveniently written as

$$
D \psi^{a_{1} \ldots a_{n}}=d \psi^{a_{1} \ldots a_{n}}+\left(n+\frac{1}{2}\right) \omega^{b} \Gamma_{b} \psi^{a_{1} \ldots a_{n}}-\omega_{b} \Gamma^{\left(a_{1}\right.} \psi^{\left.a_{2} \ldots a_{n}\right) b} .
$$

The standard supergravity action in [41-43] is then recovered for $n=0$, while the theory discussed in section 2 corresponds to $n=1$.

The generic theory can also be formulated in terms of a Chern-Simons action for a gauge field that takes values in the hyper-Poincaré algebra, given by

$$
A=e^{a} P_{a}+\omega^{a} J_{a}+\psi_{a_{1} \ldots a_{n}}^{\alpha} Q_{\alpha}^{a_{1} \ldots a_{n}} .
$$

Here $Q_{\alpha}^{a_{1} \ldots a_{n}}$ correspond to $\Gamma$-traceless fermionic generators of spin $n+\frac{1}{2}$. The explicit expression of the generic hyper-Poincaré algebra can be compactly written in terms of its Maurer-Cartan form (see appendix C). The field equations then read $F=d A+A^{2}=0$, with

$$
F=R^{a} J_{a}+\tilde{T}^{a} P_{a}+D \psi_{a_{1} \ldots a_{n}}^{\alpha} Q_{\alpha}^{a_{1} \ldots a_{n}},
$$

where the hypercovariant torsion is now given by

$$
\tilde{T}^{a}=T^{a}-\frac{1}{2}\left(n+\frac{1}{2}\right) i \bar{\psi}_{a_{1} \ldots a_{n}} \Gamma^{a} \psi^{a_{1} \ldots a_{n}} .
$$


Thus, by construction, the action is invariant under gauge transformations generated by $\lambda=\epsilon_{a_{1} \ldots a_{n}}^{\alpha} Q_{\alpha}^{a_{1} \ldots a_{n}}$, so that

$$
\begin{aligned}
\delta e^{a} & =\left(n+\frac{1}{2}\right) i \bar{\epsilon}_{a_{1} \ldots a_{n}} \Gamma^{a} \psi^{a_{1} \ldots a_{n}}, \\
\delta \omega^{a} & =0 \\
\delta \psi^{a_{1} \ldots a_{n}} & =D \epsilon^{a_{1} \ldots a_{n}} .
\end{aligned}
$$

\subsection{Killing tensor-spinors}

According to (7.6), a purely bosonic configuration is invariant under local hypersymmetry transformations provided the following "Killing tensor-spinor equation" is fulfilled:

$$
d \epsilon_{a_{1} \ldots a_{n}}+\left(n+\frac{1}{2}\right) \omega^{b} \Gamma_{b} \epsilon_{a_{1} \ldots a_{n}}-\omega^{b} \Gamma_{\left(a_{1}\right.} \epsilon_{\left.a_{2} \ldots a_{n}\right) b}=0 .
$$

Since the field equations imply the vanishing of the curvature two-form $R^{a}$, the general solution of (7.7) is now given by

$$
\epsilon_{a_{1} \cdots a_{n}}^{\alpha}=\left(g_{S}^{-1}\right)_{\beta}^{\alpha}\left(g_{V}\right)_{a_{1}}^{b_{1}} \cdots\left(g_{V}\right)_{a_{n}}^{b_{n}} \eta_{b_{1} \cdots b_{n}}^{\beta},
$$

where $g_{S}$ and $g_{V}$ are defined in eq. (3.3). As explained in section 3, both stand for the same group element $g$ that determines the spin connection, $\omega=g^{-1} d g$, but expressed in the spinor and the vector representations, respectively. In the generic case, $\eta_{b_{1} \cdots b_{n}}^{\beta}$ is a constant $\Gamma$-traceless tensor-spinor. Unbroken hypersymmetries then correspond to Killing tensor-spinors of the form (7.8), that are globally well-defined.

The hypersymmetry properties of the class of solutions discussed in section 3.1, describing cosmological spacetimes and configurations with conical defects, then go as follows. For any configuration with $\mathcal{P} \neq 0, g_{S}$ and $g_{V}$ are given by (3.8) and (3.9), respectively; while in the case of $\mathcal{P}=0$, they read as in eq. (3.10). Therefore, in the case of $\mathcal{P}>0$ the solutions cannot possess globally-defined Killing tensor-spinors, because $\epsilon_{a_{1} \cdots a_{n}}^{\alpha}$ in (7.8) do not fulfill neither periodic nor antiperiodic boundary conditions. This means that hypersymmetries are necessarily broken for cosmological spacetimes.

By virtue of (7.8) and (3.10), configurations with $\mathcal{P}=0$ only admit constant Killing tensor-spinors that fulfill the following condition:

$$
\left(n+\frac{1}{2}\right) \Gamma_{1} \epsilon_{a_{1} \ldots a_{n}}-\Gamma_{\left(a_{1}\right.} \epsilon_{\left.a_{2} \ldots a_{n}\right) 1}=0,
$$

which implies that they have a single nonvanishing component given by $\epsilon_{00 \cdots 0}^{-}=\eta_{00 \cdots 0}^{-}$. Therefore, this class of spacetimes possesses just one unbroken hypersymmetry, which relates to the fact that there is only one hypersymmetry bound that saturates for fermions with periodic boundary conditions (see below).

As explained in section 3.1, smooth solutions with conical defects are maximally hypersymmetric and their energy is determined by $\mathcal{P}=-k j^{2} / \pi<0$, where $j$ is a (half-)integer. For this class of configurations, the explicit form of the Killing tensor-spinors is then given by (7.8), with $g_{S}$ and $g_{V}$ being described by eqs. (3.12) and (3.13), respectively. It will also be shown below that conical surpluses are excluded by the hypersymmetry bounds, which are fulfilled only for $j^{2}=1 / 4$, which corresponds to the case of Minkowski spacetime. 


\subsection{Asymptotically flat structure and hypersymmetry bounds}

In order to describe the asymptotically flat behaviour of hypergravity in the generic case, it is convenient to make use of the $\Gamma$-traceless condition of the fields and the generators, which amounts to reduce the number of independent components. The hyper-Poincaré algebra in (C.2) can then be alternatively written as

$$
\begin{aligned}
& {\left[\hat{\mathcal{J}}_{m}, \hat{\mathcal{J}}_{n}\right]=(m-n) \hat{\mathcal{J}}_{m+n}} \\
& {\left[\hat{\mathcal{J}}_{m}, \hat{\mathcal{P}}_{n}\right]=(m-n) \hat{\mathcal{P}}_{m+n},} \\
& {\left[\hat{\mathcal{J}}_{m}, \hat{\mathcal{Q}}_{p}\right]=(s m-p) \hat{\mathcal{Q}}_{m+p},} \\
& \left\{\hat{\mathcal{Q}}_{p}, \hat{\mathcal{Q}}_{q}\right\}=f_{p, q}^{(s)} \hat{\mathcal{P}}_{p+q},
\end{aligned}
$$

with $m, n=0, \pm 1$, and $p, q= \pm \frac{1}{2}, \pm \frac{3}{2} \ldots, \pm s$, where $s$ stands for the spin of the fermionic generators $\hat{\mathcal{Q}}_{p}$. The structure constants fulfill $f_{p, q}^{(s)}=f_{q, p}^{(s)}=f_{-p,-q}^{(s)}$, and the nonvanishing ones are given by

$$
f_{p,-p}^{(s)}=-\frac{2 p}{s+p+1} f_{p,-p-1}^{(s)}=(-1)^{p+\frac{1}{2}} 2 p \prod_{k=\frac{1}{2}}^{|p|} \frac{(2 s+2 k)}{(2 s-2(k-1))}
$$

provided $|p+q| \leq 1$. Here the fermionic generators have been normalized according to $f_{\frac{1}{2},-\frac{1}{2}}^{(s)}=-1$.

It is amusing to verify that the Jacobi identity now translates into the fact that the structure constants $f_{p, q}^{(s)}$ solve the following recursion relation:

$$
(m-(p+q)) f_{q, p}^{(s)}-(s m-p) f_{q, m+p}^{(s)}-(s m-q) f_{p, m+q}^{(s)}=0 .
$$

For later purposes it is useful to note that

$$
f_{s,-s}^{(s)}=(-1)^{s+\frac{1}{2}} \frac{2 s}{2 s+1} \frac{(4 s) ! !}{(2 s-1) ! !^{2}} .
$$

The structure constants can also be conveniently written as

$$
f_{m, n}^{(s)}=\sum_{l=0}^{s-\frac{1}{2}} h_{m, n}^{(l)}
$$

where $h_{m, n}^{(l)}$ stand for homogeneous polynomials of degree $2 l$ in $m, n$, i. e., $h_{\lambda m, \lambda n}^{(l)}=\lambda^{2 l} h_{m, n}^{(l)}$. Indeed, as it is shown below, the asymptotic symmetry algebra can be naturally expressed in terms of $h_{m, n}^{(l)}$, where $m, n$ are extended to be arbitrary (half-)integers. Note that in the case of supergravity $f_{m, n}^{(1 / 2)}=-1$, while for fermionic generators of spin $s=3 / 2$, the form of $f_{m, n}^{(3 / 2)}$ can be read from eq. (4.2). In the case of fermionic generators with $s=5 / 2,7 / 2$ the explicit form of $f_{m, n}^{(5 / 2)}$ and $f_{m, n}^{(7 / 2)}$ is given in appendices D.2 and D.3, respectively. 
Following the lines of section 4, the asymptotic form of the gauge field can be written as in eq. (4.3), so that at a fixed time slice, the dynamical field is proposed to be given by

$$
a_{\phi}=\hat{\mathcal{J}}_{1}-\frac{\pi}{k}\left(\mathcal{J} \hat{\mathcal{P}}_{-1}+\mathcal{P} \hat{\mathcal{J}}_{-1}+\alpha_{s} \psi \hat{\mathcal{Q}}_{-s}\right)
$$

with

$$
\alpha_{s}=\left(f_{-s, s+1}^{(s)}\right)^{-1}=-2 s\left(f_{s,-s}^{(s)}\right)^{-1}
$$

and $f_{s,-s}^{(s)}$ can be read from eq. (7.13).

The asymptotic symmetries are then generically spanned by a hyper-Poincaré-valued parameter of the form

$$
\lambda=T \hat{\mathcal{P}}_{1}+Y \hat{\mathcal{J}}_{1}+\mathcal{E} \hat{\mathcal{Q}}_{s}+\eta_{(s)}[T, Y, \mathcal{E}]
$$

where $\eta_{(s)}[T, Y, \mathcal{E}]$ goes along all but the lowest weight generators, provided the fields $\mathcal{J}$, $\mathcal{P}, \psi$ transform in a suitable way.

The asymptotic form of the Lagrange multiplier can then be written in terms of the chemical potentials according to

$$
a_{u}=\lambda\left[\mu_{\mathcal{P}}, \mu_{\mathcal{J}}, \mu_{\psi}\right] .
$$

Its form is preserved under evolution in time as long as the field equations are fulfilled in the asymptotic region, and the parameters are subject to appropriate conditions, being described by first order equations in time.

In order to integrate the variation of the canonical generators in (4.11), one needs the relevant fermionic component of the invariant bilinear form, which is given by

$$
\left\langle\hat{\mathcal{Q}}_{s}, \hat{\mathcal{Q}}_{-s}\right\rangle=2 \alpha_{s}^{-1}
$$

so that the global charges in the generic case acquire the same form as in eq. (4.12), i. e.,

$$
Q[T, Y, \mathcal{E}]=-\int(T \mathcal{P}+Y \mathcal{J}-i \mathcal{E} \psi) d \phi
$$

Once expanded in Fourier modes, the nonvanishing Poisson brackets of the canonical generators are given by

$$
\begin{aligned}
& i\left\{\mathcal{J}_{m}, \mathcal{J}_{n}\right\}=(m-n) \mathcal{J}_{m+n}, \\
& i\left\{\mathcal{J}_{m}, \mathcal{P}_{n}\right\}=(m-n) \mathcal{P}_{m+n}+k m^{3} \delta_{m+n, 0}, \\
& i\left\{\mathcal{J}_{m}, \psi_{n}\right\}=(s m-n) \psi_{m+n} \\
& i\left\{\psi_{m}, \psi_{n}\right\}=\sum_{q=0}^{s-1 / 2} \frac{(-1)^{2 s-q}}{s-q+\frac{1}{2}}\left(\frac{2}{k}\right)^{s-q-\frac{1}{2}} h_{m, n}^{(q)} \mathcal{P}_{m+n}^{s-q-\frac{1}{2}}+(-1)^{s-\frac{1}{2}} \frac{2 k m^{2 s+1}}{\alpha_{s}(2 s) !} \delta_{m+n, 0}+\Xi_{m+n}^{(s)} .
\end{aligned}
$$

The conformal weight of the fermionic generators $\psi_{n}$ with respect to $\mathcal{J}_{m}$ is given by $\Delta=$ $s+1$. Here $h_{m, n}^{(q)}$ stand for the homogeneous polynomials defined through eq. (7.14), extended to the case of (half-)integers, and

$$
\mathcal{P}_{m+n}^{r}:=\sum_{i_{1}, \cdots i_{r}} \mathcal{P}_{m+n-i_{1} \cdots-i_{r}} \mathcal{P}_{i_{1}} \cdots \mathcal{P}_{i_{r}}
$$


Here $\Xi_{m+n}^{(s)}$ stands for the mode expansion of nonlinear terms that contains derivatives of $\mathcal{P}$, and becomes nontrivial provided $s>3 / 2$. Indeed, according to eqs. (D.9) and (4.13), in the case of supergravity $(s=1 / 2)$, and for $s=3 / 2$, one finds that $\Xi_{m+n}^{(1 / 2)}=\Xi_{m+n}^{(3 / 2)}=0$; while for $s=5 / 2$ it is proportional to the mode expansion of $\left(\mathcal{P}^{\prime}\right)^{2}$ (see eq. (D.20)). The explicit form of $\Xi^{(7 / 2)}$ is given in eq. (D.30).

As in section 5, the asymptotic symmetry algebra (7.21) also implies the existence of nonlinear bounds for the energy. Indeed, making the same assumptions, for $m=-n=p$, the (fermionic) anticommutator is manifestly positive-definite. Furthermore, since in the "rest frame" the bosonic global charges just correspond to $\mathcal{P}_{0}$, the nonlinear terms described by $\Xi_{m+n}^{(s)}$ in the fermionic anticommutator do not contribute. Therefore, in the generic case the bounds are given by

$$
\prod_{i=0}^{n}\left(p^{2}+(2 i+1)^{2} \frac{\pi \mathcal{P}}{k}\right) \geq 0,
$$

where $p$ is a (half-)integer for the case of fermionic fields of spin $s=n+3 / 2$ that fulfill (anti)periodic boundary conditions.

It is then clear that the bounds are fulfilled for configurations with $\mathcal{P}>0$, as it is the case of cosmological spacetimes. The fact that they never saturate agrees with the nonexistence of globally-defined Killing tensor-spinors. Note that in the case of $\mathcal{P}=0$ the bounds are also satisfied, while the one with $p=0$ saturates, which corresponds to the fact that configurations of this sort admit a single unbroken hypersymmetry, being generated by a constant Killing tensor-spinor.

For the class of maximally hypersymmetric smooth solutions with negative energy $\left(\mathcal{P}=-k j^{2} / \pi\right)$ described in section 3.1 , the bounds (7.23) read

$$
\prod_{i=0}^{n}\left(p^{2}-(2 i+1)^{2} j^{2}\right) \geq 0,
$$

which implies that the only case that fulfills all of them, also saturate the ones for $p= \pm(2 i+1) / 2$, with $i=0,1, \ldots, n$, and corresponds to $j^{2}=1 / 4$. Thus, Minkowski spacetime becomes naturally selected at the ground state in the case of fermions that satisfy antiperiodic boundary conditions, possessing the maximum number of Killing tensorspinors described by (7.8), with (3.12) and (3.13).

In sum, in the case of fermions that fulfill periodic boundary conditions the energy spectrum is nonnegative $(\mathcal{P} \geq 0)$, so that the allowed class of solutions is generically characterized by the cosmological spacetimes described in section 3.1. The ground state is then given by a configuration of vanishing energy that saturates only one of the bounds $(p=0)$. This corresponds to the null orbifold which, as shown in section 7.1, possesses a single Killing tensor-spinor. If the fermions satisfy antiperiodic boundary conditions, the spectrum becomes enlarged since the bounds now imply that $\mathcal{P} \geq-k / 4 \pi$. Nonetheless, since conical defects and surpluses generically do not fulfill the field equation in vacuum, they are discarded unless they are smooth. According to (7.24), in this case the ground state saturates as many bounds as the maximum number of Killing tensor-spinors, and it can be identified with Minkowski spacetime, so that the spectrum acquires a gap. 


\section{Final remarks}

In the case of fermionic fields of spin $s=n+3 / 2$, the locally hypersymmetric extension of the action $I_{\mu, \gamma}$ in (6.4), that includes parity-odd terms, can also be formulated as a Chern-Simons theory for the hyper-Poincaré group in (C.2). In order to carry out this task, the invariant bilinear form has to be suitably modified, so that it acquires additional components being determined by eq. (6.8). The gauge field reads

$$
A=e^{a} P_{a}+\hat{\omega}^{a} J_{a}+\psi_{a_{1} \ldots a_{n}}^{\alpha} Q_{\alpha}^{a_{1} \ldots a_{n}},
$$

where, as in section $6, \hat{\omega}^{a}=\omega^{a}+\gamma e^{a}$. Therefore, up to a boundary term, the action of the extended hypergravity theory reduces to

$$
I_{\mu, \gamma, \psi_{n}}=I_{\mu, \gamma}+\frac{k}{4 \pi} \int i \bar{\psi}_{a_{1} \ldots a_{n}}\left[D+\left(n+\frac{1}{2}\right) \gamma e^{b} \Gamma_{b}\right] \psi^{a_{1} \ldots a_{n}},
$$

being by construction locally invariant under

$$
\begin{aligned}
\delta e^{a} & =\left(n+\frac{1}{2}\right) i \bar{\epsilon}_{a_{1} \ldots a_{n}} \Gamma^{a} \psi^{a_{1} \ldots a_{n}}, \\
\delta \omega^{a} & =-\left(n+\frac{1}{2}\right) i \gamma \bar{\epsilon}_{a_{1} \ldots a_{n}} \Gamma^{a} \psi^{a_{1} \ldots a_{n}}, \\
\delta \psi^{a_{1} \ldots a_{n}} & =\left[D+\left(n+\frac{1}{2}\right) \gamma e^{b} \Gamma_{b}\right] \epsilon^{a_{1} \ldots a_{n}}-\gamma e_{b} \Gamma^{\left(a_{1}\right.} \epsilon^{\left.a_{2} \ldots a_{n}\right) b} .
\end{aligned}
$$

Note that the extended hypergravity action (8.2), and its corresponding local hypersymmetry transformations (8.3), agree with the corresponding ones for the locally hypersymmetric extension of General Relativity, given by (7.1) and (7.6), respectively, in the case of $\mu=\gamma=0$. Consequently, a suitable set of asymptotically flat boundary conditions for the extended theory is also proposed to be described by gauge fields of the form (4.3), (7.15), and (7.18). The canonical generators of the asymptotic symmetries then reduce to the ones in eq. (6.11), with $\tilde{\mathcal{J}}=\mathcal{J}+\mu \mathcal{P}$, so that their algebra is readily found to be described by (7.21), but with an additional central extension along the Virasoro subalgebra, precisely as in eq. (6.12).

It is worth pointing out that prescribing the asymptotic behaviour of gauge fields to be described by deviations with respect to a reference background that go along the highest weight generators of the algebra, turns out to be a very successful strategy. Indeed, this is not only the case of General Relativity in three spacetime dimensions [28], but it is also so for its locally supersymmetric extension with or without cosmological constant [21, 44], or even when the theory is nonminimally coupled to higher spin fields [17-20, 26, 27, 45-49].

One of the interesting features of dealing with hypersymmetry, is that nonlinear bounds for the energy have been shown to naturally emerge from the anticommutator of fermionic generators. In the case of vanishing cosmological constant, the hypersymmetry bounds for the theory in vacuum turn out to exclude solutions that describe conical defects and surpluses $[10,11]$, despite the latter are maximally (hyper)symmetric. In presence of higher spin fields, the analogue of this class of configurations has been discussed in [7, 9, 50-55]. It 
is then worth highlighting that, according to the results that have been recently obtained for hypergravity with negative cosmological constant [9], one is naturally led to expect that only a suitable subset of asymptotically flat solitonic-like solutions might fulfill the hypersymmetry bounds, for which the higher spin charges become tuned in terms of the mass. Indeed, it is amusing to verify that the gauge group $S p(4) \otimes O S p(1 \mid 4)$ admits an inequivalent Inönü-Wigner contraction as compared with the one described in section 4.1, so that the electric-like spin- 4 charges cannot be consistently decoupled in this alternative flat limit of the $(0,1)$ theory. This contraction is defined through a different change of basis: ${ }^{2}$

$$
\begin{array}{rlrl}
\hat{\mathcal{P}}_{i} & =\frac{1}{\ell}\left(\hat{\mathcal{L}}_{i}^{+}+\hat{\mathcal{L}}_{-i}^{-}\right), & \hat{\mathcal{J}}_{i}=\hat{\mathcal{L}}_{i}^{+}-\hat{\mathcal{L}}_{-i}^{-}, & \\
\hat{\mathcal{W}}_{n}=\frac{1}{\ell}\left(\hat{\mathcal{U}}_{n}^{+}+\hat{\mathcal{U}}_{-n}^{-}\right), & \hat{\mathcal{V}}_{n}=\hat{\mathcal{U}}_{n}^{+}-\hat{\mathcal{U}}_{-n}^{-}, & \hat{\mathcal{Q}}_{p}=\sqrt{\frac{6}{\ell}} \hat{\mathcal{S}}_{p}^{+},
\end{array}
$$

where $\hat{\mathcal{L}}_{i}^{-}, \hat{\mathcal{U}}_{n}^{-}$stand for the generators of the (left) $s p(4)$ algebra, and $\hat{\mathcal{L}}_{i}^{+}, \hat{\mathcal{U}}_{n}^{+}, \hat{\mathcal{S}}_{p}^{+}$span the (right) osp (1|4) algebra. Thus, in the limit of large AdS radius, $\ell \rightarrow \infty$, the nonvanishing components of the (anti)commutators of the new algebra read

$$
\begin{aligned}
& {\left[\hat{\mathcal{J}}_{i}, \hat{\mathcal{J}}_{j}\right]=(i-j) \hat{\mathcal{J}}_{i+j}, \quad\left[\hat{\mathcal{J}}_{i}, \hat{\mathcal{P}}_{j}\right]=(i-j) \hat{\mathcal{P}}_{i+j}, } {\left[\hat{\mathcal{J}}_{i}, \hat{\mathcal{V}}_{n}\right]=(3 i-n) \hat{\mathcal{V}}_{i+n}, } \\
& {\left[\hat{\mathcal{J}}_{i}, \hat{\mathcal{V}}_{n}\right]=(3 i-n) \hat{\mathcal{V}}_{i+n}, \quad\left[\hat{\mathcal{J}}_{i}, \hat{\mathcal{V}}_{n}\right]=(3 i-n) \hat{\mathcal{V}}_{i+n}, \quad\left(\frac{3 i}{2}-p\right) \hat{\mathcal{Q}}_{i+p}, } \\
& {\left[\hat{\mathcal{V}}_{m}, \hat{\mathcal{V}}_{n}\right]=} \frac{1}{2^{2} 3}(m-n)\left(\left(m^{2}+n^{2}-4\right)\left(m^{2}+n^{2}-\frac{2}{3} m n-9\right)-\frac{2}{3}(m n-6) m n\right) \hat{\mathcal{J}}_{m+n} \\
&+\frac{1}{6}(m-n)\left(m^{2}-m n+n^{2}-7\right) \hat{\mathcal{V}}_{m+n}, \\
& {\left[\hat{\mathcal{V}}_{m}, \hat{\mathcal{V}}_{n}\right]=} \frac{1}{2^{2} 3}(m-n)\left(\left(m^{2}+n^{2}-4\right)\left(m^{2}+n^{2}-\frac{2}{3} m n-9\right)-\frac{2}{3}(m n-6) m n\right) \hat{\mathcal{P}}_{m+n} \\
&+\frac{1}{6}(m-n)\left(m^{2}-m n+n^{2}-7\right) \hat{\mathcal{V}}_{m+n}, \\
& {\left[\hat{\mathcal{V}}_{m}, \hat{\mathcal{Q}}_{p}\right]=} \frac{1}{2^{3} 3}\left(2 m^{3}-8 m^{2} p+20 m p^{2}+82 p-23 m-40 p^{3}\right) \hat{\mathcal{Q}}_{m+p}, \\
&\left\{\hat{\mathcal{Q}}_{p}, \hat{\mathcal{Q}}_{q}\right\}= 3 \hat{\mathcal{V}}_{p+q}+\frac{1}{2^{2}}\left(6 p^{2}-8 p q+6 q^{2}-9\right) \hat{\mathcal{P}}_{p+q},
\end{aligned}
$$

which is to be regarded to span the gauge group of flat hypergravity coupled to spin- 4 fields. Here, $i, j=0, \pm 1, m, n=0, \pm 1, \pm 2, \pm 3$, and $p, q= \pm \frac{1}{2}, \pm \frac{3}{2}$. The mode expansion of the asymptotically flat symmetry algebra of hypergravity with a fermionic spin- $5 / 2$ field, being coupled to spin-4 fields, is then expected to be such that the nonvanishing Poisson

\footnotetext{
${ }^{2}$ Note that $O S p(1 \mid 4)$, corresponding to the super- $\mathrm{AdS}_{4}$ group, as well as the superconformal group in three spacetime dimensions, admits two interesting consistent "flat limits" $(\ell \rightarrow \infty)$, which can be obtained rescaling the generators either as in eq. (4.21), or in (8.4), provided the left copy is switched off.
} 
brackets are given by

$$
\begin{aligned}
i\left\{\mathcal{J}_{m}, \mathcal{J}_{n}\right\}= & (m-n) \mathcal{J}_{m+n}, \quad i\left\{\mathcal{J}_{m}, \mathcal{P}_{n}\right\}=(m-n) \mathcal{P}_{m+n}+k m^{3} \delta_{m+n, 0} \\
i\left\{\mathcal{J}_{m}, \mathcal{W}_{n}\right\}= & (3 m-n) \mathcal{W}_{m+n}, \quad i\left\{\mathcal{J}_{m}, \mathcal{V}_{n}\right\}=(3 m-n) \mathcal{V}_{m+n}, \\
i\left\{\mathcal{P}_{m}, \mathcal{V}_{n}\right\}= & (3 m-n) \mathcal{W}_{m+n}, \quad i\left\{\mathcal{J}_{m}, \psi_{n}\right\}=\left(\frac{3 m}{2}-n\right) \psi_{m+n}, \\
i\left\{\mathcal{V}_{m}, \mathcal{V}_{n}\right\}= & \frac{1}{2^{2} 3^{2}}(m-n)\left(3 m^{4}-2 m^{3} n+4 m^{2} n^{2}-2 m n^{3}+3 n^{4}\right) \mathcal{J}_{m+n} \\
& +\frac{1}{6}(m-n)\left(m^{2}-m n+n^{2}\right) \mathcal{V}_{m+n}-\frac{2^{3} 3 \pi}{k}(m-n) \Theta_{m+n}^{(6)} \\
& -\frac{7^{2} \pi}{3^{2} k}(m-n)\left(m^{2}+4 m n+n^{2}\right) \Theta_{m+n}^{(4)} \\
& \frac{1}{2^{2} 3^{2}}(m-n)\left(3 m^{4}-2 m^{3} n+4 m^{2} n^{2}-2 m n^{3}+3 n^{4}\right) \mathcal{P}_{m+n} \\
& +\frac{1}{6}(m-n)\left(m^{2}-m n+n^{2}\right) \mathcal{W}_{m+n}-\frac{2^{3} 3 \pi}{k}(m-n) \Omega_{m+n}^{(6)} \\
& -\frac{7^{2} \pi}{3^{2} k}(m-n)\left(m^{2}+4 m n+n^{2}\right) \Omega_{m+n}^{(4)}+\frac{k}{2^{2} 3^{2}} m^{7} \delta_{m+n, 0}, \\
\left.i \mathcal{V}_{m}, \mathcal{W}_{n}\right\} & \frac{1}{2^{2} 3}\left(m^{3}-4 m^{2} n+10 m n^{2}-20 n^{3}\right) \psi_{m+n}-\frac{23 \pi}{3 k} i \Omega_{m+n}^{(11 / 2)} \\
& +\frac{\pi}{3 k}(23 m-82 n) \Omega_{m+n}^{(9 / 2)}, \\
i\left\{\mathcal{V}_{m}, \psi_{n}\right\} & 3 \mathcal{W}_{m+n}+\frac{3}{2}\left(m^{2}-\frac{4}{3} m n+n^{2}\right) \mathcal{P}_{m+n}+\frac{9 \pi}{k} \Omega_{m+n}^{(4)}+k m^{4} \delta_{m+n, 0},
\end{aligned}
$$

where $\Omega_{m}^{(l)}$, and $\Theta_{m}^{(l)}$ stand for the mode expansion of the following nonlinear terms: ${ }^{3}$

$$
\begin{aligned}
\Omega^{(4)} & =\frac{1}{2} \mathcal{P}^{2} \\
\Theta^{(4)} & =\mathcal{J} \mathcal{P} \\
\Omega^{(9 / 2)} & =\frac{1}{2} \mathcal{P} \psi \\
\Omega^{(11 / 2)} & =\frac{27}{46} \mathcal{P}^{\prime} \psi \\
\Omega^{(6)} & =-\frac{7}{36} \mathcal{W} \mathcal{P}-\frac{2 \pi}{3 k} \mathcal{P}^{3}+\frac{295}{864}\left(\mathcal{P}^{\prime}\right)^{2}+\frac{11}{27} \mathcal{P} \mathcal{P}^{\prime \prime}, \\
\Theta^{(6)} & =-\frac{7}{36}(\mathcal{V} \mathcal{P}+\mathcal{W} \mathcal{J})-\frac{2 \pi}{k} \mathcal{P}^{2} \mathcal{J}+\frac{295}{432} \mathcal{J}^{\prime} \mathcal{P}^{\prime}+\frac{11}{27}\left(\mathcal{J P}^{\prime \prime}+\mathcal{P} \mathcal{J}^{\prime \prime}\right)+\frac{25}{72} i \psi \psi^{\prime}
\end{aligned}
$$

Indeed, this asymptotic symmetry algebra is recovered from a contraction that corresponds to a different flat limit of $\mathrm{W}_{(2,4)} \oplus \mathrm{W}_{\left(2, \frac{5}{2}, 4\right)}$, as compared with the one in 4.1. The flat limit

${ }^{3}$ The infinite-dimensional nonlinear algebras in eqs. (4.22) and (8.6), correspond to different hypersymmetric extensions of the $\mathrm{BMS}_{3}$ algebra, being isomorphic to the Galilean conformal algebra in two dimensions, and then relevant in the context of non-relativistic holography [16, 56-59]. 
is now defined according to

$$
\begin{aligned}
& \mathcal{P}_{n}=\frac{1}{\ell}\left(\mathcal{L}_{n}^{+}+\mathcal{L}_{-n}^{-}\right), \quad \mathcal{J}_{n}=\mathcal{L}_{n}^{+}-\mathcal{L}_{-n}^{-}, \\
& \mathcal{W}_{n}=\frac{1}{\ell}\left(\mathcal{U}_{n}^{+}+\mathcal{U}_{-n}^{-}\right), \quad \mathcal{V}_{n}=\mathcal{U}_{n}^{+}-\mathcal{U}_{-n}^{-}, \quad \psi_{n}=\sqrt{\frac{6}{\ell}} \Psi_{n}^{+},
\end{aligned}
$$

where the level also rescales as $\kappa=k \ell$.

Moreover, once the modes $\mathcal{P}_{m}$ are shifted according to (4.14), it is simple to verify that the wedge algebra of (8.6) reduces to the algebra of the gauge group in (8.5).

From the anticommutator of the fermionic generators in (8.6), one then finds that the zero modes of the energy and the electric-like spin- 4 charge, $2 \pi \mathcal{P}=\mathcal{P}_{0}, 2 \pi \mathcal{W}=\mathcal{W}_{0}$, fulfill the following bounds

$$
3 \mathcal{W}+\frac{9 \pi}{2 k} \mathcal{P}^{2}+5 p^{2} \mathcal{P}+\frac{k}{2 \pi} p^{4} \geq 0
$$

which agree with the bounds in [9] in the flat limit. It would then be interesting to explore different classes of solutions endowed with electric-like spin-4 charge, including cosmological spacetimes and solitonic-like configurations that fulfill the bounds (8.9), as well as the hypersymmetric ones that should saturate them. Note that since the bounds (8.9) factorize as

$$
\left(p^{2}+\lambda_{[+]}^{2}\right)\left(p^{2}+\lambda_{[-]}^{2}\right) \geq 0
$$

it is natural to expect that the eigenvalues of the holonomy of the dynamical gauge field $a_{\phi}$ along an angular cycle, for the class of solutions aforementioned, have to be given by

$$
\lambda_{[ \pm]}^{2}=\frac{5 \pi}{k}\left(\mathcal{P} \pm \frac{4}{5} \sqrt{\mathcal{P}^{2}-\frac{3 k}{8 \pi} \mathcal{W}}\right)
$$

In the case of solitonic-like solutions, these eigenvalues should then correspond to a couple of purely imaginary integers, that become related for the class of configurations that fulfill the bounds (8.10), saturating just some of them.

As a final remark, since the hyper-Poincaré group actually exists for $d \geq 3$ spacetime dimensions [6], it would be interesting to explore whether similar results as the ones obtained here could extend to higher-dimensional spacetimes. In this sense, despite the no-go results in four dimensions [2,3,60-62], some interesting results have been recently found in the case of hypergravity at the noninteracting level [63]. Whether these results correspond to a suitable weak field limit of Vasiliev higher spin gravity $[64,65]$, or another theory that has yet to be found, remains as an open question.

\section{Acknowledgments}

We thank M. Henneaux, A. Pérez and D. Tempo for helpful discussions. We also thank the International Solvay Institutes and the ULB for warm hospitality. O.F. and R.T. wish to thank Amilcar de Queiroz, Emanuele Orazi, and the organizers of the school and conference "Theoretical Frontiers in Black Holes and Cosmology", hosted by IIPUFRN, during June 2015, for the opportunity of presenting this work. O.F. thanks Conicyt 
for financial support. This research has been partially supported by Fondecyt grants № 1130658, 1121031, 3150448. The Centro de Estudios Científicos (CECs) is funded by the Chilean Government through the Centers of Excellence Base Financing Program of Conicyt.

\section{A Conventions}

We follow the conventions of [6]. The orientation is chosen to be such that the Levi-Civita symbol fulfills $\varepsilon_{012}=1$, and the Minkowski metric is assumed to be non-diagonal, whose only nonvanishing components read $\eta_{01}=\eta_{10}=\eta_{22}=1$. Round brackets correspond to symmetrization of the indices enclosed by them, so that

$$
X^{(a \mid} Y^{b} Z^{\mid c)}=X^{a} Y^{b} Z^{c}+X^{c} Y^{b} Z^{a} .
$$

Note that the three-dimensional Dirac matrices, that satisfy the Clifford algebra $\left\{\Gamma_{a}, \Gamma_{b}\right\}=$ $2 \eta_{a b}$, fulfill the following identity:

$$
\Gamma^{a} \Gamma^{b} \Gamma^{c}=\varepsilon^{a b c}+\eta^{a b} \Gamma^{c}+\eta^{b c} \Gamma^{a}-\eta^{a c} \Gamma^{b} .
$$

The generators of the Lorentz group, in the spinorial and vector (adjoint) representations, are assumed to be given by $\left(J_{a}\right)_{\beta}^{\alpha}=\frac{1}{2}\left(\Gamma_{a}\right)_{\beta}^{\alpha}$, and $\left(J_{a}\right)_{c}^{b}=-\varepsilon_{a}^{b}{ }_{c}$, respectively.

The three-dimensional $\Gamma$-matrices are chosen as

$$
\Gamma_{0}=\frac{1}{\sqrt{2}}\left(\sigma_{1}+i \sigma_{2}\right), \quad \Gamma_{1}=\frac{1}{\sqrt{2}}\left(\sigma_{1}-i \sigma_{2}\right), \quad \Gamma_{2}=\sigma_{3},
$$

where $\sigma_{i}$ stand for the Pauli matrices:

$$
\sigma_{1}=\left(\begin{array}{ll}
0 & 1 \\
1 & 0
\end{array}\right), \quad \sigma_{2}=\left(\begin{array}{cc}
0 & -i \\
i & 0
\end{array}\right), \quad \sigma_{3}=\left(\begin{array}{cc}
1 & 0 \\
0 & -1
\end{array}\right) .
$$

For a vector-spinor $\psi_{a}^{\alpha}$, with $\alpha=+,-$, and $a=0,1,2$, the Majorana conjugate is defined as $\bar{\psi}_{\alpha a}=\psi_{a}^{\beta} C_{\beta \alpha}$, where the charge conjugation matrix $C$, and its inverse are chosen as

$$
C_{\alpha \beta}=\left(\begin{array}{cc}
0 & -1 \\
1 & 0
\end{array}\right), \quad C^{\alpha \beta}=\left(\begin{array}{cc}
0 & 1 \\
-1 & 0
\end{array}\right)
$$

so that $C^{T}=-C$, and $\left(C \Gamma_{a}\right)^{T}=C \Gamma_{a}$.

\section{B Killing vector-spinors from an alternative approach}

Killing vector-spinors $\epsilon_{a}^{\alpha}$ in (3.2) that are globally well defined can be recovered as a particular case of the asymptotic symmetries discussed in section 4 . Indeed, they are of the form $\epsilon_{a}^{\alpha} Q_{\alpha}^{a}=\lambda[0,0, \mathcal{E}]$, with $\lambda$ given by (4.5). Hence, for the class of bosonic configurations discussed in section 3.1, that only carries the zero modes of the global charges, 
and their corresponding chemical potentials are constant, the components of the Killing vector-spinors can be written as

$$
\lambda[0,0, \mathcal{E}]=\mathcal{E} \hat{\mathcal{Q}}_{\frac{3}{2}}-\mathcal{E}^{\prime} \hat{\mathcal{Q}}_{\frac{1}{2}}-\frac{1}{2}\left(\frac{3 \pi}{k} \mathcal{E} \mathcal{P}-\mathcal{E}^{\prime \prime}\right) \hat{\mathcal{Q}}_{-\frac{1}{2}}-\frac{\pi}{3 k}\left(-\frac{7}{2} \mathcal{E}^{\prime} \mathcal{P}+\frac{k}{2 \pi} \mathcal{E}^{\prime \prime \prime}\right) \hat{\mathcal{Q}}_{-\frac{3}{2}} .
$$

The requirements of invariance under hypersymmetry can then be read from eqs. (4.7), (4.10), so that the Killing vector-spinor equation reduces to

$$
\begin{aligned}
\delta \psi & =-\frac{9 \pi}{2 k} \mathcal{P}^{2} \mathcal{E}+5 \mathcal{P} \mathcal{E}^{\prime \prime}-\frac{k}{2 \pi} \mathcal{E}^{\prime \prime \prime \prime}=0, \\
\dot{\mathcal{E}} & =\mu_{\mathcal{J}} \mathcal{E}^{\prime}
\end{aligned}
$$

which can be readily integrated. In fact, the solution of eq. (B.2) is generically given by

$$
\mathcal{E}=\mathcal{A}_{1} e^{\sqrt{\frac{\pi \mathcal{P}}{k}} \phi}+\mathcal{A}_{2} e^{-\sqrt{\frac{\pi \mathcal{P}}{k}} \phi}+\mathcal{A}_{3} e^{3 \sqrt{\frac{\pi \mathcal{P}}{k}} \phi}+\mathcal{A}_{4} e^{-3 \sqrt{\frac{\pi \mathcal{P}}{k}} \phi}
$$

where $\mathcal{A}_{I}=\mathcal{A}_{I}(u)$ stand for four arbitrary functions.

In the case of $\mathcal{P}>0, \mathcal{E}$ clearly cannot fulfill neither antiperiodic nor periodic boundary conditions, and therefore, cosmological spacetimes break all the hypersymmetries.

Note that if the energy vanishes $(\mathcal{P}=0)$, eq. (B.2) integrates in a different way:

$$
\mathcal{E}=\mathcal{A}_{0}+\mathcal{A}_{1} \phi+\mathcal{A}_{2} \phi^{2}+\mathcal{A}_{3} \phi^{3}
$$

Periodicity then implies that $\mathcal{E}=\mathcal{A}_{0}(u)$, while the remaining equation (B.3) fixes the arbitrary function to be a constant. Hence, vanishing energy configurations, as the null orbifold, admit a single constant Killing vector-spinor.

Finally, for $\mathcal{P}=-k n^{2} / \pi<0$, eq. (B.4) reads

$$
\mathcal{E}=\mathcal{A}_{1} e^{i n \phi}+\mathcal{A}_{1}^{*} e^{-i n \phi}+\mathcal{A}_{3} e^{3 i n \phi}+\mathcal{A}_{3}^{*} e^{-3 i n \phi},
$$

so that $n$ turns out to be a (half-)integer for fermions fulfilling (anti)periodic boundary conditions. The remaining equation (B.3) then fixes the form of the arbitrary functions $\mathcal{A}_{I}(u)$, and hence there are four independent Killing vector-spinors, determined by

$$
\mathcal{E}=\mathcal{E}_{1} e^{i n\left(\mu_{\mathcal{J}}^{u+\phi)}\right.}+\mathcal{E}_{1}^{*} e^{-i n\left(\mu_{\mathcal{J}} u+\phi\right)}+\mathcal{E}_{3} e^{3 i n\left(\mu_{\mathcal{J}}^{u+\phi)}\right.}+\mathcal{E}_{3}^{*} e^{-3 i n\left(\mu_{\mathcal{J}} u+\phi\right)} .
$$

It is worth pointing out that Minkowski spacetime, which corresponds to $n^{2}=1 / 4$, is the only one of this class that fulfills all the hypersymmetry bounds (5.3), saturating precisely four of them in the case of antiperiodic boundary conditions. Indeed, the remaining solutions of this sort, in spite of possessing the maximum number of hypersymmetries, become manifestly excluded by the bounds. In the case of periodic boundary conditions, the null orbifold also satisfies the bounds, but it saturates only one of them. 


\section{Hyper-Poincaré algebra with fermionic generators of spin $n+\frac{1}{2}$}

The extension of the Poincaré algebra in the generic case includes fermionic tensor-spinor generators $Q_{\alpha}^{a_{1} \ldots a_{n}}$, being completely symmetric in the vector indices, and $\Gamma$-traceless, i. e., $Q^{a_{1} \ldots a_{n}} \Gamma_{a_{1}}=0[6]$. The Maurer-Cartan 1-form,

$$
\Omega=\rho^{a} P_{a}+\tau^{a} J_{a}+\chi_{a_{1} \ldots a_{n}}^{\alpha} Q_{\alpha}^{a_{1} \ldots a_{n}},
$$

corresponds to a flat connection that fulfills

$$
\begin{aligned}
d \tau^{a} & =-\frac{1}{2} \epsilon^{a b c} \tau_{b} \tau_{c}, \\
d \rho^{a} & =-\epsilon^{a b c} \tau_{b} \rho_{c}+\frac{1}{2}\left(n+\frac{1}{2}\right) i \bar{\chi}_{a_{1} \ldots a_{n}} \Gamma^{a} \chi^{a_{1} \ldots a_{n}}, \\
d \chi^{a_{1} \ldots a_{n}} & =-\left(n+\frac{1}{2}\right) \tau^{b} \Gamma_{b} \chi^{a_{1} \ldots a_{n}}+\tau_{b} \Gamma^{\left(a_{1} \mid\right.} \chi^{\left.\mid a_{2} \ldots a_{n}\right) b},
\end{aligned}
$$

where $\chi_{a_{1} \ldots a_{n}}$ is $\Gamma$-traceless, and completely symmetric in the vector indices. Apart from $I_{1}=P^{a} P_{a}$, this algebra admits an additional Casimir operator given by

$$
I_{2}=2 J^{a} P_{a}+Q_{\alpha a_{1} \ldots a_{n}} C^{\alpha \beta} Q_{\beta}^{a_{1} \ldots a_{n}} .
$$

\section{Asymptotic hypersymmetry algebra}

\section{D.1 Spin-3/2 fields (supergravity)}

In our conventions, the super-Poincaré algebra with $\mathcal{N}=1$ reads

$$
\begin{aligned}
{\left[J_{a}, J_{b}\right] } & =\varepsilon_{a b c} J^{c}, \\
{\left[J_{a}, P_{b}\right] } & =\varepsilon_{a b c} P^{c}, \\
{\left[J_{a}, Q_{\alpha}\right] } & =\frac{1}{2}\left(\Gamma_{a}\right)_{\alpha}^{\beta} Q_{\beta b}, \\
\left\{Q_{\alpha}, Q_{\beta}\right\} & =-\frac{1}{2}\left(C \Gamma^{c}\right)_{\alpha \beta} P_{c},
\end{aligned}
$$

so that changing the basis according to

$$
\begin{array}{lll}
\hat{\mathcal{J}}_{-1}=-2 J_{0}, & \hat{\mathcal{J}}_{1}=J_{1}, & \hat{\mathcal{J}}_{0}=J_{2}, \\
\hat{\mathcal{P}}_{-1}=-2 P_{0}, & \hat{\mathcal{P}}_{1}=P_{1}, & \hat{\mathcal{P}}_{0}=P_{2}, \\
\hat{\mathcal{Q}}_{-\frac{1}{2}}=2^{\frac{3}{4}} Q_{+}, & \hat{\mathcal{Q}}_{\frac{1}{2}}=2^{\frac{1}{4}} Q_{-}, &
\end{array}
$$

it acquires the following form

$$
\begin{aligned}
& {\left[\hat{\mathcal{J}}_{m}, \hat{\mathcal{J}}_{n}\right]=(m-n) \hat{\mathcal{J}}_{m+n},} \\
& {\left[\hat{\mathcal{J}}_{m}, \hat{\mathcal{P}}_{n}\right]=(m-n) \hat{\mathcal{P}}_{m+n},} \\
& {\left[\hat{\mathcal{J}}_{m}, \hat{\mathcal{Q}}_{p}\right]=\left(\frac{m}{2}-p\right) \hat{\mathcal{Q}}_{m+p},} \\
& \left\{\hat{\mathcal{Q}}_{p}, \hat{\mathcal{Q}}_{q}\right\}=-\hat{\mathcal{P}}_{p+q} .
\end{aligned}
$$

with $m, n=0, \pm 1$, and $p, q= \pm \frac{1}{2}$. 
The asymptotic form of the dynamical gauge field then reads

$$
a_{\phi}=\hat{\mathcal{J}}_{1}-\frac{\pi}{k}\left(\mathcal{P} \hat{\mathcal{J}}_{-1}+\mathcal{J} \hat{\mathcal{P}}_{-1}+\psi \hat{\mathcal{Q}}_{-\frac{1}{2}}\right),
$$

which is mapped into itself under gauge transformations generated by

$$
\lambda=T \hat{\mathcal{P}}_{1}+Y \hat{\mathcal{J}}_{1}+\mathcal{E} \hat{\mathcal{Q}}_{\frac{1}{2}}+\eta_{\left(\frac{1}{2}\right)}[T, Y, \mathcal{E}]
$$

with

$$
\begin{aligned}
\eta_{\left(\frac{1}{2}\right)}[T, Y, \mathcal{E}]= & -T^{\prime} \hat{\mathcal{P}}_{0}-Y^{\prime} \hat{\mathcal{J}}_{0}-\left(\mathcal{E}^{\prime}+\frac{\pi Y \psi}{k}\right) \hat{\mathcal{Q}}_{-\frac{1}{2}}+\frac{1}{2}\left(Y^{\prime \prime}-\frac{2 \pi Y \mathcal{P}}{k}\right) \hat{\mathcal{J}}_{-1} \\
& +\frac{1}{2}\left(T^{\prime \prime}-\frac{2 \pi T \mathcal{P}}{k}-\frac{2 \pi Y \mathcal{J}}{k}+\frac{i \pi \mathcal{E} \psi}{k}\right) \hat{\mathcal{P}}_{-1}
\end{aligned}
$$

provided the fields transform according to:

$$
\begin{aligned}
& \delta \mathcal{P}=2 \mathcal{P} Y^{\prime}+\mathcal{P}^{\prime} Y-\frac{k}{2 \pi} Y^{\prime \prime \prime} \\
& \delta \mathcal{J}=2 \mathcal{J} Y^{\prime}+\mathcal{J}^{\prime} Y+2 \mathcal{P} T^{\prime}+\mathcal{P}^{\prime} T-\frac{k}{2 \pi} T^{\prime \prime \prime}+\frac{3}{2} i \psi \mathcal{E}^{\prime}+\frac{1}{2} i \psi^{\prime} \mathcal{E} \\
& \delta \psi=\frac{3}{2} \psi Y^{\prime}+\psi^{\prime} Y-\mathcal{P} \mathcal{E}+\frac{k}{\pi} \mathcal{E}^{\prime \prime} .
\end{aligned}
$$

Once expanded in modes, the asymptotic symmetry algebra is found to be given by

$$
\begin{aligned}
i\left\{\mathcal{J}_{m}, \mathcal{J}_{n}\right\} & =(m-n) \mathcal{J}_{m+n}, \\
i\left\{\mathcal{J}_{m}, \mathcal{P}_{n}\right\} & =(m-n) \mathcal{P}_{m+n}+k m^{3} \delta_{m+n, 0}, \\
i\left\{\mathcal{J}_{m}, \psi_{n}\right\} & =\left(\frac{m}{2}-n\right) \psi_{m+n} \\
i\left\{\psi_{m}, \psi_{n}\right\} & =\mathcal{P}_{m+n}+2 k m^{2} \delta_{m+n, 0}
\end{aligned}
$$

and hence in the case of fermions that fulfill periodic boundary conditions, the energy $\mathcal{P}=\frac{\mathcal{P}_{0}}{2 \pi}$ is bounded to be nonnegative,

$$
\mathcal{P} \geq 0
$$

while in the case of fermions subject to antiperiodic boundary conditions, the energy fulfills

$$
\frac{1}{4}+\frac{\pi \mathcal{P}}{k} \geq 0
$$

These results agree with the ones in [21].

\section{D.2 Spin-7/2 fields}

In the case of fermionic generators of (conformal) spin $\Delta=7 / 2$, the hyper-Poincaré algebra can be written as

$$
\begin{aligned}
& {\left[\hat{\mathcal{J}}_{m}, \hat{\mathcal{J}}_{n}\right]=(m-n) \hat{\mathcal{J}}_{m+n},} \\
& {\left[\hat{\mathcal{J}}_{m}, \hat{\mathcal{P}}_{n}\right]=(m-n) \hat{\mathcal{P}}_{m+n},} \\
& {\left[\hat{\mathcal{J}}_{m}, \hat{\mathcal{Q}}_{p}\right]=\left(\frac{5 m}{2}-p\right) \hat{\mathcal{Q}}_{m+p},} \\
& \left\{\hat{\mathcal{Q}}_{p}, \hat{\mathcal{Q}}_{q}\right\}=f_{p, q}^{(5 / 2)} \hat{\mathcal{P}}_{p+q}
\end{aligned}
$$


with

$$
\begin{aligned}
f_{p, q}^{(5 / 2)}= & -\frac{1}{192}\left[80\left(p^{4}+q^{4}\right)-128\left(p^{3} q+p q^{3}\right)\right. \\
& \left.+144 p^{2} q^{2}-620\left(p^{2}+q^{2}\right)+832 p q+675\right]
\end{aligned}
$$

where $m, n=0, \pm 1$, and $p, q= \pm \frac{1}{2}, \pm \frac{3}{2}, \pm \frac{5}{2}$.

The asymptotic behaviour of the dynamical gauge field now reads

$$
a_{\phi}=\hat{\mathcal{J}}_{1}-\frac{\pi}{k}\left(\mathcal{J} \hat{\mathcal{P}}_{-1}+\mathcal{P} \hat{\mathcal{J}}_{-1}+\frac{\psi}{10} \hat{\mathcal{Q}}_{-\frac{5}{2}}\right)
$$

so that the asymptotic symmetries are now spanned by

$$
\lambda=T \hat{\mathcal{P}}_{1}+Y \hat{\mathcal{J}}_{1}+\mathcal{E} \hat{\mathcal{Q}}_{\frac{1}{2}}+\eta_{\left(\frac{5}{2}\right)}[T, Y, \mathcal{E}]
$$

with

$$
\begin{aligned}
\eta_{\left(\frac{5}{2}\right)}[T, Y, \mathcal{E}]= & -T^{\prime} \hat{\mathcal{P}}_{0}-Y^{\prime} \hat{\mathcal{J}}_{0}-\mathcal{E}^{\prime} \hat{\mathcal{Q}}_{\frac{3}{2}}+\frac{1}{2}\left(T^{\prime \prime}-\frac{2 \pi T \mathcal{P}}{k}-\frac{2 \pi Y \mathcal{J}}{k}-\frac{5 i \pi \mathcal{E} \psi}{k}\right) \hat{\mathcal{P}}_{-1} \\
& +\frac{1}{2}\left(Y^{\prime \prime}-\frac{2 \pi Y \mathcal{P}}{k}\right) \hat{\mathcal{J}}_{-1}+\frac{1}{2}\left(\mathcal{E}^{\prime \prime}-\frac{5 \pi \mathcal{E} \mathcal{P}}{k}\right) \hat{\mathcal{Q}}_{\frac{1}{2}} \\
& -\frac{1}{6}\left(\mathcal{E}^{\prime \prime \prime}-\frac{13 \pi \mathcal{E}^{\prime} \mathcal{P}}{k}-\frac{5 \pi \mathcal{E} \mathcal{P}^{\prime}}{k}\right) \hat{\mathcal{Q}}_{-\frac{1}{2}} \\
& +\frac{1}{24}\left(\mathcal{E}^{(4)}+\frac{45 \pi^{2} \mathcal{E} \mathcal{P}^{2}}{k^{2}}-\frac{22 \pi \mathcal{E}^{\prime \prime} \mathcal{P}}{k}-\frac{18 \pi \mathcal{E}^{\prime} \mathcal{P}^{\prime}}{k}-\frac{5 \pi \mathcal{E} \mathcal{P}^{\prime \prime}}{k}\right) \hat{\mathcal{Q}}_{-\frac{3}{2}} \\
& -\frac{1}{120}\left(\mathcal{E}^{(5)}+\frac{149 \pi^{2} \mathcal{E}^{\prime} \mathcal{P}^{2}}{k^{2}}+\frac{12 \pi Y \psi}{k}-\frac{30 \pi \mathcal{E}^{\prime \prime \prime} \mathcal{P}}{k}\right. \\
& \left.+\frac{130 \pi^{2} \mathcal{E} \mathcal{P} \mathcal{P}^{\prime}}{k^{2}}-\frac{40 \pi \mathcal{E}^{\prime \prime} \mathcal{P}^{\prime}}{k}-\frac{23 \pi \mathcal{E}^{\prime} \mathcal{P}^{\prime \prime}}{k}-\frac{5 \pi \mathcal{E} \mathcal{P}^{\prime \prime \prime}}{k}\right) \hat{\mathcal{Q}}_{-\frac{5}{2}}
\end{aligned}
$$

and the transformation law of the fields is given by

$$
\begin{aligned}
\delta \mathcal{P}= & 2 \mathcal{P} Y^{\prime}+\mathcal{P}^{\prime} Y-\frac{k}{2 \pi} Y^{\prime \prime \prime} \\
\delta \mathcal{J}= & 2 \mathcal{J} Y^{\prime}+\mathcal{J}^{\prime} Y+2 \mathcal{P} T^{\prime}+\mathcal{P}^{\prime} T-\frac{k}{2 \pi} T^{\prime \prime \prime}+\frac{7}{2} i \psi \mathcal{E}^{\prime}+\frac{5}{2} i \psi^{\prime} \mathcal{E} \\
\delta \psi= & \frac{7}{2} \psi Y^{\prime}+\psi^{\prime} Y+\left(-\frac{75 \pi^{2} \mathcal{P}^{3}}{4 k^{2}}+\frac{65 \pi\left(\mathcal{P}^{\prime}\right)^{2}}{6 k}+\frac{155 \pi \mathcal{P} \mathcal{P}^{\prime \prime}}{12 k}-\frac{5}{12} \mathcal{P}^{(4)}\right) \mathcal{E} \\
& +\left(\frac{259 \pi \mathcal{P} \mathcal{P}^{\prime}}{6 k}-\frac{7}{3} \mathcal{P}^{\prime \prime \prime}\right) \mathcal{E}^{\prime}+\left(\frac{259 \pi \mathcal{P}^{2}}{12 k}-\frac{21}{4} \mathcal{P}^{\prime \prime}\right) \mathcal{E}^{\prime \prime}-\frac{35}{6} \mathcal{E}^{\prime \prime \prime} \mathcal{P}^{\prime}-\frac{35}{12} \mathcal{E}^{(4)} \mathcal{P}+\frac{k \mathcal{E}^{(6)}}{12 \pi}
\end{aligned}
$$


The Poisson brackets of the asymptotic symmetry algebra in this case read

$$
\begin{aligned}
i\left\{\mathcal{J}_{m}, \mathcal{J}_{n}\right\}= & (m-n) \mathcal{J}_{m+n} \\
i\left\{\mathcal{J}_{m}, \mathcal{P}_{n}\right\}= & (m-n) \mathcal{P}_{m+n}+k m^{3} \delta_{m+n, 0} \\
i\left\{\mathcal{J}_{m}, \psi_{n}\right\}= & \left(\frac{5 m}{2}-n\right) \psi_{m+n} \\
i\left\{\psi_{m}, \psi_{n}\right\}= & \frac{1}{12}\left(5 m^{4}-8 m^{3} n+9 m^{2} n^{2}-8 m n^{3}+5 n^{4}\right) \mathcal{P}_{m+n} \\
& +\frac{1}{48 k}\left(155 m^{2}-208 m n+155 n^{2}\right) \sum_{q} \mathcal{P}_{m+n-q} \mathcal{P}_{q} \\
& +\frac{75}{16 k^{2}} \sum_{q} \mathcal{P}_{m+n-q-r} \mathcal{P}_{q} \mathcal{P}_{r}+\frac{k}{6} m^{6} \delta_{m+n, 0}+\Xi_{m+n}^{(5 / 2)}
\end{aligned}
$$

where $\Xi_{m}^{(5 / 2)}=\int \Xi^{(5 / 2)} e^{-i m \phi} d \phi$ stands for the mode expansion of

$$
\Xi^{(5 / 2)}=\frac{25 \pi\left(\mathcal{P}^{\prime}\right)^{2}}{12 k}
$$

The anticommutator of the fermionic charges then implies that the energy has to fulfill the following bounds

$$
\left(p^{2}+\frac{25 \pi \mathcal{P}}{k}\right)\left(p^{2}+\frac{9 \pi \mathcal{P}}{k}\right)\left(p^{2}+\frac{\pi \mathcal{P}}{k}\right) \geq 0,
$$

with $p$ given by a (half-)integer for fermions that fulfill (anti)periodic boundary conditions.

\section{D.3 Spin-9/2 fields}

The hyper-Poincaré algebra with fermionic generators of (conformal) spin $\Delta=9 / 2$ is described by

$$
\begin{aligned}
{\left[\hat{\mathcal{J}}_{m}, \hat{\mathcal{J}}_{n}\right] } & =(m-n) \hat{\mathcal{J}}_{m+n}, \\
{\left[\hat{\mathcal{J}}_{m}, \hat{\mathcal{P}}_{n}\right] } & =(m-n) \hat{\mathcal{P}}_{m+n}, \\
{\left[\hat{\mathcal{J}}_{m}, \hat{\mathcal{Q}}_{p}\right] } & =\left(\frac{7 m}{2}-p\right) \hat{\mathcal{Q}}_{m+p}, \\
\left\{\hat{\mathcal{Q}}_{p}, \hat{\mathcal{Q}}_{q}\right\} & =f_{p, q}^{(7 / 2)} \hat{\mathcal{P}}_{p+q}
\end{aligned}
$$

where

$$
\begin{aligned}
f_{p, q}^{(7 / 2)}= & \frac{1}{2304}\left[112\left(p^{6}+q^{6}\right)-192\left(p^{5} q+p q^{5}\right)+240\left(p^{4} q^{2}+p^{2} q^{4}\right)-256 p^{3} q^{3}\right. \\
& -2240\left(p^{4}+q^{4}\right)+3616\left(p^{3} q+p q^{3}\right)-4080 p^{2} q^{2} \\
& \left.+11578\left(p^{2}+q^{2}\right)-15592 p q-11025\right]
\end{aligned}
$$

and with $m, n=0, \pm 1$, and $p, q= \pm \frac{1}{2}, \pm \frac{3}{2}, \pm \frac{5}{2}, \pm \frac{7}{2}$.

The asymptotic fall-off of the dynamical gauge field is now given by

$$
a_{\phi}=\hat{\mathcal{J}}_{1}-\frac{\pi}{k}\left(\mathcal{J} \hat{\mathcal{P}}_{-1}+\mathcal{P} \hat{\mathcal{J}}_{-1}-\frac{\psi}{35} \hat{\mathcal{Q}}_{-\frac{7}{2}}\right)
$$


and the asymptotic symmetries turn out to be parametrized according to

$$
\lambda=T \hat{\mathcal{P}}_{1}+Y \hat{\mathcal{J}}_{1}+\mathcal{E} \hat{\mathcal{Q}}_{\frac{1}{2}}+\eta_{\left(\frac{7}{2}\right)}[T, Y, \mathcal{E}]
$$

with

$$
\begin{aligned}
& \eta_{\left(\frac{7}{2}\right)}[T, Y, \mathcal{E}]=-T^{\prime} \hat{\mathcal{P}}_{0}-Y^{\prime} \hat{\mathcal{J}}_{0}-\mathcal{E}^{\prime} \hat{\mathcal{Q}}_{\frac{5}{2}}+\frac{1}{2}\left(T^{\prime \prime}-\frac{2 \pi T \mathcal{P}}{k}-\frac{2 \pi Y \mathcal{J}}{k}-\frac{7 i \pi \mathcal{E} \psi}{k}\right) \hat{\mathcal{P}}_{-1} \\
& +\frac{1}{2}\left(Y^{\prime \prime}-\frac{2 \pi Y \mathcal{P}}{k}\right) \hat{\mathcal{J}}_{-1}+\frac{1}{2}\left(\mathcal{E}^{\prime \prime}-\frac{7 \pi \mathcal{E} \mathcal{P}}{k}\right) \hat{\mathcal{Q}}_{\frac{3}{2}}-\frac{1}{6}\left(\mathcal{E}^{\prime \prime \prime}-\frac{19 \pi \mathcal{E}^{\prime} \mathcal{P}}{k}-\frac{7 \pi \mathcal{E} \mathcal{P}^{\prime}}{k}\right) \hat{\mathcal{Q}}_{\frac{1}{2}} \\
& +\frac{1}{24}\left(\mathcal{E}^{(4)}+\frac{105 \pi^{2} \mathcal{E} \mathcal{P}^{2}}{k^{2}}-\frac{34 \pi \mathcal{E}^{\prime \prime} \mathcal{P}}{k}-\frac{26 \pi \mathcal{E}^{\prime} \mathcal{P}^{\prime}}{k}-\frac{7 \pi \mathcal{E} \mathcal{P}^{\prime \prime}}{k}\right) \hat{\mathcal{Q}}_{-\frac{1}{2}} \\
& -\frac{1}{120}\left(\mathcal{E}^{(5)}+\frac{409 \pi^{2} \mathcal{E}^{\prime} \mathcal{P}^{2}}{k^{2}}+\frac{322 \pi^{2} \mathcal{E} \mathcal{P} \mathcal{P}^{\prime}}{k^{2}}-\frac{50 \pi \mathcal{E}^{\prime \prime \prime} \mathcal{P}}{k}-\frac{60 \pi \mathcal{E}^{\prime \prime} \mathcal{P}^{\prime}}{k}\right. \\
& \left.-\frac{33 \pi \mathcal{E}^{\prime} \mathcal{P}^{\prime \prime}}{k}-\frac{7 \pi \mathcal{E} \mathcal{P}^{\prime \prime \prime}}{k}\right) \hat{\mathcal{Q}}_{-\frac{3}{2}}+\frac{1}{720}\left(\mathcal{E}^{(6)}-\frac{1575 \pi^{3} \mathcal{E} \mathcal{P}^{3}}{k^{3}}\right. \\
& +\frac{919 \pi^{2} \mathcal{E}^{\prime \prime} \mathcal{P}^{2}}{k^{2}}+\frac{1530 \pi^{2} \mathcal{E}^{\prime} \mathcal{P} \mathcal{P}^{\prime}}{k^{2}}+\frac{427 \pi^{2} \mathcal{E} \mathcal{P} \mathcal{P}^{\prime \prime}}{k^{2}}+\frac{322 \pi^{2} \mathcal{E}\left(\mathcal{P}^{\prime}\right)^{2}}{k^{2}} \\
& \left.-\frac{65 \pi \mathcal{E}^{(4)} \mathcal{P}}{k}-\frac{110 \pi \mathcal{E}^{(3)} \mathcal{P}^{\prime}}{k}-\frac{93 \pi \mathcal{E}^{\prime \prime} \mathcal{P}^{\prime \prime}}{k}-\frac{40 \pi \mathcal{E}^{\prime} \mathcal{P}^{\prime \prime \prime}}{k}-\frac{7 \pi \mathcal{E} \mathcal{P}^{(4)}}{k}\right) \hat{\mathcal{Q}}_{-\frac{5}{2}} \\
& -\frac{1}{5040}\left(\mathcal{E}^{(7)}-\frac{6483 \pi^{3} \mathcal{E}^{\prime \prime} \mathcal{P}^{3}}{k^{3}}-\frac{8589 \pi^{3} \mathcal{E} \mathcal{P}^{2} \mathcal{P}^{\prime}}{k^{3}}+\frac{1519 \pi^{2} \mathcal{E}^{\prime \prime \prime} \mathcal{P}^{2}}{k^{2}}\right. \\
& +\frac{4088 \pi^{2} \mathcal{E}^{\prime \prime} \mathcal{P} \mathcal{P}^{\prime}}{k^{2}}+\frac{2353 \pi^{2} \mathcal{E}^{\prime} \mathcal{P} \mathcal{P}^{\prime \prime}}{k^{2}}+\frac{1852 \pi^{2} \mathcal{E}^{\prime}\left(\mathcal{P}^{\prime}\right)^{2}}{k^{2}} \\
& +\frac{511 \pi^{2} \mathcal{E} \mathcal{P} \mathcal{P}^{\prime \prime \prime}}{k^{2}}+\frac{1071 \pi^{2} \mathcal{E} \mathcal{P}^{\prime} \mathcal{P}^{\prime \prime}}{k^{2}}-\frac{144 \pi Y \psi}{k}-\frac{77 \pi \mathcal{E}^{(5)} \mathcal{P}}{k}-\frac{175 \pi \mathcal{E}^{(4)} \mathcal{P}^{\prime}}{k} \\
& \left.-\frac{203 \pi \mathcal{E}^{\prime \prime \prime} \mathcal{P}^{\prime \prime}}{k}-\frac{133 \pi \mathcal{E}^{\prime \prime} \mathcal{P}^{\prime \prime \prime}}{k}-\frac{47 \pi \mathcal{E}^{\prime} \mathcal{P}^{(4)}}{k}-\frac{7 \pi \mathcal{E} \mathcal{P}^{(5)}}{k}\right) \hat{\mathcal{Q}}_{-\frac{7}{2}},
\end{aligned}
$$

so that the fields transform as

$$
\begin{aligned}
\delta \mathcal{P}= & 2 \mathcal{P} Y^{\prime}+\mathcal{P}^{\prime} Y-\frac{k}{2 \pi} Y^{\prime \prime \prime}, \\
\delta \mathcal{J}= & 2 \mathcal{J} Y^{\prime}+\mathcal{J}^{\prime} Y+2 \mathcal{P} T^{\prime}+\mathcal{P}^{\prime} T-\frac{k}{2 \pi} T^{\prime \prime \prime}+\frac{9}{2} i \psi \mathcal{E}^{\prime}+\frac{7}{2} i \psi^{\prime} \mathcal{E}, \\
\delta \psi= & \frac{9}{2} \psi Y^{\prime}+\psi^{\prime} Y+\left(-\frac{1225 \pi^{3} \mathcal{P}^{4}}{16 k^{3}}+\frac{5789 \pi^{2} \mathcal{P}^{\prime \prime} \mathcal{P}^{2}}{72 k^{2}}+\frac{2429 \pi^{2}\left(\mathcal{P}^{\prime}\right)^{2} \mathcal{P}}{18 k^{2}}\right. \\
& \left.-\frac{35 \pi \mathcal{P}^{(4)} \mathcal{P}}{9 k}-\frac{119 \pi\left(\mathcal{P}^{\prime \prime}\right)^{2}}{16 k}-\frac{791 \pi \mathcal{P}^{\prime} \mathcal{P}^{\prime \prime \prime}}{72 k}+\frac{7}{144} \mathcal{P}^{(6)}\right) \mathcal{E}+ \\
& +\left(\frac{3229 \pi^{2} \mathcal{P}^{\prime} \mathcal{P}^{2}}{12 k^{2}}-\frac{131 \pi \mathcal{P}^{\prime \prime \prime} \mathcal{P}}{6 k}-\frac{99 \pi \mathcal{P}^{\prime} \mathcal{P}^{\prime \prime}}{2 k}+\frac{3}{8} \mathcal{P}^{(5)}\right) \mathcal{E}^{\prime}
\end{aligned}
$$




$$
\begin{aligned}
& +\left(\frac{3229 \pi^{2} \mathcal{P}^{3}}{36 k^{2}}-\frac{197 \pi \mathcal{P}^{\prime \prime} \mathcal{P}}{4 k}-\frac{165 \pi\left(\mathcal{P}^{\prime}\right)^{2}}{4 k}+\frac{5}{4} \mathcal{P}^{(4)}\right) \mathcal{E}^{\prime \prime} \\
& +\left(\frac{7}{3} \mathcal{P}^{\prime \prime \prime}-\frac{329 \pi \mathcal{P} \mathcal{P}^{\prime}}{6 k}\right) \mathcal{E}^{(3)}+\left(\frac{21}{8} \mathcal{P}^{\prime \prime}-\frac{329 \pi \mathcal{P}^{2}}{24 k}\right) \mathcal{E}^{(4)} \\
& +\frac{7}{4} \mathcal{E}^{(5)} \mathcal{P}^{\prime}+\frac{7}{12} \mathcal{E}^{(6)} \mathcal{P}-\frac{k \mathcal{E}^{(8)}}{144 \pi}
\end{aligned}
$$

The mode expansion of the asymptotic symmetry algebra is then given by

$$
\begin{aligned}
i\left\{\mathcal{J}_{m}, \mathcal{J}_{n}\right\}= & (m-n) \mathcal{J}_{m+n}, \\
i\left\{\mathcal{J}_{m}, \mathcal{P}_{n}\right\}= & (m-n) \mathcal{P}_{m+n}+k m^{3} \delta_{m+n, 0} \\
i\left\{\mathcal{J}_{m}, \psi_{n}\right\}= & \left(\frac{7 m}{2}-n\right) \psi_{m+n}, \\
i\left\{\psi_{m}, \psi_{n}\right\}= & \frac{1}{144}\left(7 m^{6}-12 m^{5} n+15 m^{4} n^{2}-16 m^{3} n^{3}+15 m^{2} n^{4}-12 m n^{5}+7 n^{6}\right) \mathcal{P}_{m+n} \\
& +\frac{1}{144 k}\left(140 m^{4}-226 m^{3} n+255 m^{2} n^{2}-226 m n^{3}+140 n^{4}\right) \sum_{q} \mathcal{P}_{m+n-q} \mathcal{P}_{q} \\
& +\frac{1}{864 k^{2}}\left(5789 m^{2}-7796 m n+5789 n^{2}\right) \sum_{q, r} \mathcal{P}_{m+n-q-r} \mathcal{P}_{q} \mathcal{P}_{r} \\
& +\frac{1225}{128 k^{3}} \sum_{q, r, t} \mathcal{P}_{m+n-q-r} \mathcal{P}_{q} \mathcal{P}_{r} \mathcal{P}_{t}+\frac{k}{72} m^{8} \delta_{m+n, 0}+\Xi_{m+n}^{(7 / 2)},
\end{aligned}
$$

where

$$
\Xi_{m+n}^{(7 / 2)}=7 \Theta_{m+n}+\left(329 m^{2}-494 m n+329 n^{2}\right) \chi_{m+n},
$$

and $\Theta_{m}$ and $\chi_{m}$ correspond to the mode expansion of

$$
\Theta=\frac{1596 \pi^{2} \mathcal{P}\left(\mathcal{P}^{\prime}\right)^{2}}{432 k^{2}}+\frac{661 \pi\left(\mathcal{P}^{\prime \prime}\right)^{2}}{432 k}, \quad \chi=\frac{\pi\left(\mathcal{P}^{\prime}\right)^{2}}{144 k},
$$

respectively.

The energy is then found to fulfill the following bounds

$$
\left(p^{2}+\frac{49 \pi \mathcal{P}}{k}\right)\left(p^{2}+\frac{25 \pi \mathcal{P}}{k}\right)\left(p^{2}+\frac{9 \pi \mathcal{P}}{k}\right)\left(p^{2}+\frac{\pi \mathcal{P}}{k}\right) \geq 0
$$

where according to the (anti)periodicity conditions for the fermions, $p$ corresponds to a (half-)integer.

Open Access. This article is distributed under the terms of the Creative Commons Attribution License (CC-BY 4.0), which permits any use, distribution and reproduction in any medium, provided the original author(s) and source are credited. 


\section{References}

[1] S. Weinberg, Photons and Gravitons in s Matrix Theory: Derivation of Charge Conservation and Equality of Gravitational and Inertial Mass, Phys. Rev. 135 (1964) B1049 [INSPIRE].

[2] C. Aragone and S. Deser, Consistency Problems of Hypergravity, Phys. Lett. B 86 (1979) 161 [InSPIRE].

[3] F.A. Berends, J.W. van Holten, P. van Nieuwenhuizen and B. de Wit, On Spin 5/2 Gauge Fields, Phys. Lett. B 83 (1979) 188 [Erratum ibid. 84B (1979) 529] [INSPIRE].

[4] S. Weinberg and E. Witten, Limits on Massless Particles, Phys. Lett. B 96 (1980) 59 [INSPIRE].

[5] C. Aragone and S. Deser, Hypersymmetry in D = 3 of Coupled Gravity Massless Spin 5/2 System, Class. Quant. Grav. 1 (1984) L9 [INSPIRE].

[6] O. Fuentealba, J. Matulich and R. Troncoso, Extension of the Poincaré group with half-integer spin generators: hypergravity and beyond, JHEP 09 (2015) 003 [arXiv: 1505.06173] [INSPIRE].

[7] B. Chen, J. Long and Y.-N. Wang, Conical Defects, Black Holes and Higher Spin (Super-)Symmetry, JHEP 06 (2013) 025 [arXiv: 1303.0109] [INSPIRE].

[8] Yu. M. Zinoviev, Hypergravity in AdS $S_{3}$, Phys. Lett. B 739 (2014) 106 [arXiv:1408.2912] [INSPIRE].

[9] M. Henneaux, A. Perez, D. Tempo and R. Troncoso, Hypersymmetry bounds and three-dimensional higher-spin black holes, JHEP 08 (2015) 021 [arXiv:1506.01847] [INSPIRE].

[10] S. Deser, R. Jackiw and G. 't Hooft, Three-Dimensional Einstein Gravity: Dynamics of Flat Space, Annals Phys. 152 (1984) 220 [inSPIRE].

[11] S. Deser and R. Jackiw, Three-Dimensional Cosmological Gravity: Dynamics of Constant Curvature, Annals Phys. 153 (1984) 405 [INSPIRE].

[12] K. Ezawa, Transition amplitude in $(2+1)$-dimensional Chern-Simons gravity on a torus, Int. J. Mod. Phys. A 9 (1994) 4727 [hep-th/9305170] [INSPIRE].

[13] L. Cornalba and M.S. Costa, A New cosmological scenario in string theory, Phys. Rev. D 66 (2002) 066001 [hep-th/0203031] [INSPIRE].

[14] L. Cornalba and M.S. Costa, Time dependent orbifolds and string cosmology, Fortsch. Phys. 52 (2004) 145 [hep-th/0310099] [INSPIRE].

[15] G. Barnich, Entropy of three-dimensional asymptotically flat cosmological solutions, JHEP 10 (2012) 095 [arXiv: 1208.4371] [INSPIRE].

[16] A. Bagchi, S. Detournay, R. Fareghbal and J. Simón, Holography of 3D Flat Cosmological Horizons, Phys. Rev. Lett. 110 (2013) 141302 [arXiv:1208.4372] [INSPIRE].

[17] M. Gary, D. Grumiller, M. Riegler and J. Rosseel, Flat space (higher spin) gravity with chemical potentials, JHEP 01 (2015) 152 [arXiv:1411.3728] [INSPIRE].

[18] J. Matulich, A. Perez, D. Tempo and R. Troncoso, Higher spin extension of cosmological spacetimes in 3D: asymptotically flat behaviour with chemical potentials and thermodynamics, JHEP 05 (2015) 025 [arXiv: 1412.1464] [INSPIRE]. 
[19] M. Henneaux, A. Perez, D. Tempo and R. Troncoso, Chemical potentials in three-dimensional higher spin anti-de Sitter gravity, JHEP 12 (2013) 048 [arXiv:1309.4362] [INSPIRE].

[20] C. Bunster, M. Henneaux, A. Perez, D. Tempo and R. Troncoso, Generalized Black Holes in Three-dimensional Spacetime, JHEP 05 (2014) 031 [arXiv: 1404.3305] [INSPIRE].

[21] G. Barnich, L. Donnay, J. Matulich and R. Troncoso, Asymptotic symmetries and dynamics of three-dimensional flat supergravity, JHEP 08 (2014) 071 [arXiv:1407.4275] [INSPIRE].

[22] O. Coussaert and M. Henneaux, Supersymmetry of the $(2+1)$ black holes, Phys. Rev. Lett. 72 (1994) 183 [hep-th/9310194] [INSPIRE].

[23] A. Ashtekar, J. Bicak and B.G. Schmidt, Asymptotic structure of symmetry reduced general relativity, Phys. Rev. D 55 (1997) 669 [gr-qc/9608042] [InSPIRE].

[24] G. Barnich and G. Compere, Classical central extension for asymptotic symmetries at null infinity in three spacetime dimensions, Class. Quant. Grav. 24 (2007) F15 [gr-qc/0610130] [INSPIRE].

[25] G. Barnich and C. Troessaert, Aspects of the BMS/CFT correspondence, JHEP 05 (2010) 062 [arXiv: 1001.1541] [INSPIRE].

[26] H. Afshar, A. Bagchi, R. Fareghbal, D. Grumiller and J. Rosseel, Spin-3 Gravity in Three-Dimensional Flat Space, Phys. Rev. Lett. 111 (2013) 121603 [arXiv:1307.4768] [INSPIRE].

[27] H.A. Gonzalez, J. Matulich, M. Pino and R. Troncoso, Asymptotically flat spacetimes in three-dimensional higher spin gravity, JHEP 09 (2013) 016 [arXiv:1307.5651] [INSPIRE].

[28] O. Coussaert, M. Henneaux and P. van Driel, The Asymptotic dynamics of three-dimensional Einstein gravity with a negative cosmological constant, Class. Quant. Grav. 12 (1995) 2961 [gr-qc/9506019] [INSPIRE].

[29] G.T. Horowitz and A.R. Steif, Singular string solutions with nonsingular initial data, Phys. Lett. B 258 (1991) 91 [InSPIRE].

[30] T. Regge and C. Teitelboim, Role of Surface Integrals in the Hamiltonian Formulation of General Relativity, Annals Phys. 88 (1974) 286 [INSPIRE].

[31] J.M. Figueroa-O'Farrill, S. Schrans and K. Thielemans, On the Casimir algebra of B(2), Phys. Lett. B 263 (1991) 378 [INSPIRE].

[32] S. Bellucci, S. Krivonos and A.S. Sorin, Linearizing W(2,4) and WB(2) algebras, Phys. Lett. B 347 (1995) 260 [hep-th/9411168] [INSPIRE].

[33] A. Achucarro and P.K. Townsend, A Chern-Simons Action for Three-Dimensional anti-de Sitter Supergravity Theories, Phys. Lett. B 180 (1986) 89 [InSPIRE].

[34] S. Deser and C. Teitelboim, Supergravity Has Positive Energy, Phys. Rev. Lett. 39 (1977) 249 [InSPIRE].

[35] C. Teitelboim, Surface Integrals as Symmetry Generators in Supergravity Theory, Phys. Lett. B 69 (1977) 240 [INSPIRE].

[36] E. Witten, A Simple Proof of the Positive Energy Theorem, Commun. Math. Phys. 80 (1981) 381 [InSPIRE].

[37] L.F. Abbott and S. Deser, Stability of Gravity with a Cosmological Constant, Nucl. Phys. B 195 (1982) 76 [inSPIRE]. 
[38] C.M. Hull, The Positivity of Gravitational Energy and Global Supersymmetry, Commun. Math. Phys. 90 (1983) 545 [InSPIRE].

[39] C. Teitelboim, Manifestly Positive Energy Expression In Classical Gravity: Simplified Derivation From Supergravity, Phys. Rev. D 29 (1984) 2763 [INSPIRE].

[40] A. Giacomini, R. Troncoso and S. Willison, Three-dimensional supergravity reloaded, Class. Quant. Grav. 24 (2007) 2845 [hep-th/0610077] [INSPIRE].

[41] S. Deser and J.H. Kay, Topologically massive supergravity, Phys. Lett. B 120 (1983) 97 [INSPIRE].

[42] S. Deser, Cosmological Topological Supergravity, in Quantum Theory Of Gravity, S.M. Christensen eds., CRC Press, Boca Raton U.S.A. (1984), pg. 374.

[43] N. Marcus and J.H. Schwarz, Three-Dimensional Supergravity Theories, Nucl. Phys. B 228 (1983) 145 [inSPIRE].

[44] M. Henneaux, L. Maoz and A. Schwimmer, Asymptotic dynamics and asymptotic symmetries of three-dimensional extended AdS supergravity, Annals Phys. 282 (2000) 31 [hep-th/9910013] [INSPIRE].

[45] M. Henneaux and S.-J. Rey, Nonlinear $W_{\infty}$ as Asymptotic Symmetry of Three-Dimensional Higher Spin Anti-de Sitter Gravity, JHEP 12 (2010) 007 [arXiv:1008.4579] [INSPIRE].

[46] A. Campoleoni, S. Fredenhagen, S. Pfenninger and S. Theisen, Asymptotic symmetries of three-dimensional gravity coupled to higher-spin fields, JHEP 11 (2010) 007 [arXiv: 1008.4744] [INSPIRE].

[47] M. Henneaux, G. Lucena Gómez, J. Park and S.-J. Rey, Super- $W_{\infty}$ Asymptotic Symmetry of Higher-Spin AdS S Supergravity, JHEP 06 (2012) 037 [arXiv:1203.5152] [INSPIRE].

[48] M. Gutperle, E. Hijano and J. Samani, Lifshitz black holes in higher spin gravity, JHEP 04 (2014) 020 [arXiv:1310.0837] [INSPIRE].

[49] M. Gutperle and Y. Li, Higher Spin Lifshitz Theory and Integrable Systems, Phys. Rev. D 91 (2015) 046012 [arXiv:1412.7085] [INSPIRE].

[50] A. Castro, R. Gopakumar, M. Gutperle and J. Raeymaekers, Conical Defects in Higher Spin Theories, JHEP 02 (2012) 096 [arXiv:1111.3381] [INSPIRE].

[51] S. Datta and J.R. David, Supersymmetry of classical solutions in Chern-Simons higher spin supergravity, JHEP 01 (2013) 146 [arXiv:1208.3921] [INSPIRE].

[52] A. Campoleoni, T. Prochazka and J. Raeymaekers, A note on conical solutions in $3 D$ Vasiliev theory, JHEP 05 (2013) 052 [arXiv: 1303.0880] [INSPIRE].

[53] A. Campoleoni and S. Fredenhagen, On the higher-spin charges of conical defects, Phys. Lett. B 726 (2013) 387 [arXiv:1307.3745] [INSPIRE].

[54] W. Li, F.-L. Lin and C.-W. Wang, Modular Properties of 3D Higher Spin Theory, JHEP 12 (2013) 094 [arXiv: 1308.2959] [INSPIRE].

[55] J. Raeymaekers, Quantization of conical spaces in 3D gravity, JHEP 03 (2015) 060 [arXiv: 1412.0278] [INSPIRE].

[56] A. Bagchi and R. Gopakumar, Galilean Conformal Algebras and AdS/CFT, JHEP 07 (2009) 037 [arXiv: 0902.1385] [inSPIRE]. 
[57] A. Bagchi, Correspondence between Asymptotically Flat Spacetimes and Nonrelativistic Conformal Field Theories, Phys. Rev. Lett. 105 (2010) 171601 [INSPIRE].

[58] A. Bagchi, S. Detournay, D. Grumiller and J. Simon, Cosmic Evolution from Phase Transition of Three-Dimensional Flat Space, Phys. Rev. Lett. 111 (2013) 181301 [arXiv: 1305.2919] [INSPIRE].

[59] A. Bagchi, R. Basu, D. Grumiller and M. Riegler, Entanglement entropy in Galilean conformal field theories and flat holography, Phys. Rev. Lett. 114 (2015) 111602 [arXiv: 1410.4089] [INSPIRE].

[60] C. Aragone and S. Deser, Higher Spin Vierbein Gauge Fermions and Hypergravities, Nucl. Phys. B 170 (1980) 329 [InSPIRE].

[61] M. Porrati, Universal Limits on Massless High-Spin Particles, Phys. Rev. D 78 (2008) 065016 [arXiv:0804.4672] [INSPIRE].

[62] X. Bekaert, N. Boulanger and P. Sundell, How higher-spin gravity surpasses the spin two barrier: no-go theorems versus yes-go examples, Rev. Mod. Phys. 84 (2012) 987 [arXiv: 1007.0435] [INSPIRE].

[63] C. Bunster, M. Henneaux, S. Hörtner and A. Leonard, Supersymmetric electric-magnetic duality of hypergravity, Phys. Rev. D 90 (2014) 045029 [arXiv: 1406.3952] [INSPIRE].

[64] M.A. Vasiliev, Consistent equation for interacting gauge fields of all spins in (3+1)-dimensions, Phys. Lett. B 243 (1990) 378 [INSPIRE].

[65] V.E. Didenko and E.D. Skvortsov, Elements of Vasiliev theory, arXiv:1401.2975 [INSPIRE]. 